University of Nebraska - Lincoln

DigitalCommons@University of Nebraska - Lincoln

8-18-2008

\title{
Threshold effects on plateau electron angular distributions in above-threshold detachment
}

Katarzyna Krajewska

University of Warsaw, Poland, kkraj@fuw.edu.pl

Ilya I. Fabrikant

University of Nebraska-Lincoln, ifabrikant@unl.edu

Anthony F. Starace

University of Nebraska-Lincoln, astarace1@unl.edu

Follow this and additional works at: https://digitalcommons.unl.edu/physicsstarace

Part of the Physics Commons

Krajewska, Katarzyna; Fabrikant, Ilya I.; and Starace, Anthony F., "Threshold effects on plateau electron angular distributions in above-threshold detachment" (2008). Anthony F. Starace Publications. 146. https://digitalcommons.unl.edu/physicsstarace/146

This Article is brought to you for free and open access by the Research Papers in Physics and Astronomy at DigitalCommons@University of Nebraska - Lincoln. It has been accepted for inclusion in Anthony F. Starace Publications by an authorized administrator of DigitalCommons@University of Nebraska - Lincoln. 


\title{
Threshold effects on plateau electron angular distributions in above-threshold detachment
}

\author{
K. Krajewska, ${ }^{1,2, *}$ Ilya I. Fabrikant, ${ }^{1}$ and Anthony F. Starace ${ }^{1}$ \\ ${ }^{1}$ Department of Physics and Astronomy, University of Nebraska, Lincoln, Nebraska 68588-0111, USA \\ ${ }^{2}$ Institute of Theoretical Physics, University of Warsaw, Hoża 69, 00-681 Warszawa, Poland \\ (Received 9 May 2008; revised manuscript received 15 July 2008; published 15 August 2008)
}

\begin{abstract}
Rates of above-threshold detachment (ATD) of $\mathrm{H}^{-}$(s-electron) and $\mathrm{F}^{-}$( $p$-electron) ions, calculated using the Sturmian-Floquet quasienergy approach, are investigated as a function of intensity for the case that the laser field intensity passes across ponderomotively shifted detachment thresholds. Pronounced enhancements of both detached electron angular distributions and fixed-angle ATD spectra in the low- and mid-energy plateau region are found at near-threshold laser intensities. These enhancements are sensitive to the initial state symmetry (i.e., the $s$ or $p$ symmetry) of the valence electron and occur in a narrow angular interval that is far from the polarization axis at electron energies near the onset of the plateau and moves toward the polarization axis with increasing electron energy. Although enhancements in detached electron energy distributions have not been observed for energies near the cutoff of the ATD plateau, the corresponding electron angular distributions are found to change shape dramatically as a function of intensity near the closing of ponderomotively shifted detachment thresholds. A comparison of our quantum-mechanically calculated angular distributions and those predicted classically in the high-energy region of the ATD plateau is presented and discussed.
\end{abstract}

DOI: $10.1103 /$ PhysRevA.78.023407

PACS number(s): $32.80 . \mathrm{Rm}, 32.80 . \mathrm{Gc}$

\section{INTRODUCTION}

A key feature of the interaction of an intense laser field with an atomic system is the so-called "plateau" in abovethreshold ionization (ATI) and above-threshold detachment (ATD) spectra, i.e., a weak dependence of the ionization (or detachment) rates on the number, $n$, of photons absorbed, over a large range of $n$ (for a recent review, see [1]). The plateau feature is known to originate from rescattering of the active electron from the remaining atomic core under the influence of the driving laser field. It extends over a range of ionized or detached electron energies, from approximately $2 U_{p}$ to approximately $10 U_{p}$, where $U_{p}$ is the ponderomotive energy corresponding to the quiver motion of an electron in a laser field.

Several experiments have demonstrated significant enhancements of the strong field plateaus, i.e., that small changes in laser intensity can result in an order of magnitude increase of ATI electrons having a range of energies at the lower end of the ATI plateau. These enhancements are still not completely understood theoretically. The experimental measurements [2-7] have been carried out for neutral atoms. The theoretical interpretations identify different possible origins of the phenomenon [4-6,8-20]. Moreover, many of the theoretical investigations $[10-12,15-17,19,20]$ are actually for the process of above-threshold detachment, since the theoretical results for ATD predict the same kind of enhancement of ATD plateau electrons with small changes of laser intensity as is found experimentally for ATI plateau electrons.

In this paper we focus on the ATD energy spectra at fixed electron detachment angle and on the angular distributions of ATD electrons as functions of laser intensity in the vicinity of ATD plateau enhancements. We find that near the onset of

\footnotetext{
*Katarzyna.Krajewska@fuw.edu.pl
}

the plateau, enhancements of the ATD rates occur over a narrow angular interval that is far from the laser polarization axis. With increasing electron detachment angle, this angular interval moves closer to the laser polarization axis. At even higher energies, near the plateau cutoff, although there is no longer any enhancement of the ATD rates, the angular distributions change shape in the vicinity of those laser intensities that result in ATD enhancements at lower energies. Since the subjects of ATI and ATD plateau enhancements as well as of ATI and ATD angular distributions have been studied by many authors, we review briefly in what follows the main works relevant to the results presented in this paper.

\section{A. Experimental and theoretical investigations of ATI and ATD plateau enhancements}

Enhancements of the high-energy plateau in both ATI and ATD have been studied in a number of experimental and theoretical papers [2-20]. Experimental measurements for rare gas atoms [2-7] have shown that the low- to mid-energy portion of the ATI plateau can be raised significantly (up to an order of magnitude) when the laser field intensity changes by only a few percent. A number of distinct theoretical interpretations of such intensity-selective enhancement of the yield of plateau electrons have been put forward.

The various theoretical interpretations may be divided into two main groups. It has been suggested in Refs. $[4,5,8,9,13,14,18]$ that the experimentally observed enhancements may be related to multiphoton resonances with ponderomotively shifted Rydberg $[4,5,8,9,18]$ or light-induced states $[13,14]$. However, the same plateau enhancements have been found theoretically for model systems that have only one bound state (and thus no excited states), such as the zero-range potential model system [6,10-12], which is appropriate for describing negative ions having a valence electron in an $s$ state. On the basis of such model calculations $[6,10-12]$, the plateau enhancements are found to coincide 
with the ponderomotive potential-induced closing of a multiphoton channel. This channel-closing (CC) scenario has both quasiclassical and quantum explanations of the calculated plateau enhancements. The quasiclassical explanation $[6,10,12]$ relates the plateau enhancement to the coherent superposition of the amplitudes for a large number of electron trajectories when a channel closing occurs. The quantum explanation [11] relates the plateau enhancements to wellknown threshold phenomena in multichannel problems at the closing of a particular channel: Specifically, the singular behavior of the cross section in a channel that is closing [21] is transmitted by multichannel interactions to other open channels [22], with the largest enhancements occurring in those open channels having the strongest interaction with the channel that is closing [17]. (The fact that the observed and calculated enhancements occur only on the ATI or ATD plateau is consistent with this quantum interpretation owing to the fact that plateau electrons originate from rescattering from the core and thus have the opportunity to have strong multichannel interactions with the channel that is closing.) Very recently, the channel-closing scenario for ATD plateau enhancements has drawn further support from calculations for multiphoton detachment of a negative ion having a valence electron in a $p$-state $[15-17,19]$. It has been shown that the ATD plateau enhancements are sensitive to the initial state symmetry, with the most pronounced enhancements occurring for an initial state having $s$ or $p$ symmetry in the case of even- or odd-photon channel closings, respectively. These results of quantum-mechanical studies have been confirmed recently by a detailed semiclassical, quantum orbit theory analysis [20]. In the context of these recent findings in support of a channel-closing scenario for ATD plateau enhancements $[6,10-12,15-17,19,20]$, it is of interest to investigate the behavior of the detached electron angular distributions as well as the fixed-angle ATD energy distributions in the vicinity of the thresholds for multiphoton transitions. This is the focus of the present work. First, however, we review very briefly prior work concerning ATD electron angular distributions.

\section{B. Experimental and theoretical investigations of ATD electron angular distributions}

A fruitful way to gain insight into the dynamics of atomic systems interacting with a strong laser field is to investigate the angular distributions (ADs) of the decay products. For the case of ATD, experiments have been particularly difficult owing to the small binding energy of the negative ion targets and the high laser intensities required to observe ATD, i.e., the detachment transition may reach saturation before the laser pulse intensity becomes sufficient to produce ATD plateau electrons. For this reason, the experiments on ATD photoelectron angular distributions of $\mathrm{H}^{-}$for the case of linearly polarized laser light [23-27] as well as corresponding theoretical studies (see, e.g., Refs. [28-30]) have focused mainly on low-order processes. Experimental investigations of ATD photoelectron angular distributions for the $\mathrm{F}^{-}$ion, which has a much larger electron affinity, were able to observe ATD electrons over a much larger range in energy [31-33], includ- ing for the case of circularly polarized light [32]. The corresponding theoretical analyses of ATD for the $\mathrm{F}^{-}$ion [34-36] showed that the experimental ATD data [25] extended up to the beginning of the ATD plateau (see, e.g., Fig. 1 of Ref. [35] and, especially, the inset figure in Fig. 2(a) of Ref. [36]).

The lowest-energy peaks in the ATD electron energy spectrum correspond to electrons that are directly detached and that do not rescatter from the atomic core. For these electrons, the angular distributions have been described well within a model that ignores final state interaction of the active electron with the atomic core $[37,38]$. Results of such a theoretical model were found to compare reasonably well with the recent (relative) experimental data for both $\mathrm{H}^{-}$detachment [25-27] and $\mathrm{F}^{-}$detachment [31-33]. For the case of two-photon detachment of $\mathrm{H}^{-}$, Telnov and $\mathrm{Chu}$ [29] compared their nonperturbative Floquet results for the detached electron angular distributions with the Keldysh-type results of Ref. [38]. Although the ADs agreed qualitatively in shape, there were significant quantitative differences [29].

In order to describe the ATD electron distribution in both energy and angle over a large range of energies, including in the region of the ATD plateau, the theoretical description must include final-state interaction of the active electron with the atomic core. In order to describe the experimental measurements for multiphoton detachment of $\mathrm{F}^{-}$[31], Frolov et al. [34,36] generalized the well-known effective-range theory for a weakly bound electron in a short-range potential to include the nonperturbative interaction of the active electron with both the short-range potential and a timedependent, harmonically varying field (e.g., the field of a laser). By comparison of the ATD spectrum for a negative ion having a valence $p$ electron $\left(\mathrm{F}^{-}\right)$with that for a negative ion having a valence $s$ electron $\left(\mathrm{H}^{-}\right)$, it was shown that the ADs for a $p$ electron are more localized around the laser field polarization direction and, also, have a more pronounced side-lobe structure near the onset of the ATD plateau [34,36].

Regarding the intensity dependence of a low-energy electron AD in the vicinity of the closure of its particular ATD channel, for the case of linearly polarized light it has been found that the angular distribution changes dramatically as the detached electron energy tends to zero, e.g., from being peaked along the polarization direction of the laser field to being isotropic just before the channel closure $[17,19,29]$. For $\mathrm{H}^{-}$(which has an $s$-state valence electron, $\ell=0$ ), such dramatic changes occur in the detached electron AD at the closure of an even-photon channel, while for $\mathrm{F}^{-}$(which has a $p$-state valence electron, $\ell=1$ ) similar dramatic changes occur at the closure of an odd-photon channel. These results may be understood on the basis of electric dipole selection rules (which specify the allowed electron partial waves) and the Wigner threshold law [21] (which specifies the nearthreshold energy dependence of each allowed partial wave amplitude). For the case of elliptically polarized light, we note that the behavior of the low-energy detached electron ADs near ATD channel closings is even more dramatic [39]; our considerations in this paper, however, are confined to the case of linearly polarized light.

\section{Focus and organization of this paper}

The focus of this paper is on the intensity dependence (in both electron energy and angle) of ATD rates for both $\mathrm{H}^{-}$and 
$\mathrm{F}^{-}$for the case of linear laser polarization. That is, we investigate how ATD electron energy and angular distributions vary with laser intensity as low-energy detached electron channels become closed. In contrast to the prior work described above, we investigate primarily the effect of channel closings on ADs for electrons on the ATD plateau, i.e., having energies greater than approximately $2 U_{p}$.

In Sec. II we present briefly the theoretical formulation upon which our fully nonperturbative, quantum results are based. We also review briefly some results of a classical analysis $[40,41]$ that are used in the interpretation of our results. In Sec. III we present and discuss in detail the angular distributions of high-energy plateau electrons, including angle-fixed ATD electron spectra in the vicinity of channel closings. Also, comparisons between some of our exact results and those predicted classically are presented. In Sec. IV we summarize our results and present some conclusions.

\section{THEORETICAL FORMULATION}

As in Refs. [17,19], we treat the problem of intense laser detachment of the $\mathrm{H}^{-}$and $\mathrm{F}^{-}$negative ions by solving a single-active-electron (SAE) model essentially exactly. The active electron is assumed to be bound in a Yukawa potential and the laser field is assumed to be monochromatic. The time-dependent Schrödinger equation is solved using the Floquet approach for the complex quasienergy of the system. In Sec. II A we introduce key notations and parameters. In Sec. II B we review the basic equations of the Floquet approach. Finally, in Sec. II C we review briefly some basic expressions arising from a classical analysis of laser ionization $[40,41]$ that we employ in Sec. III in the interpretation of our quantum numerical results. Throughout this paper, atomic units (a.u.) are used unless otherwise specified.

\section{A. Key notation and parameters}

The laser field is described by a linearly polarized plane wave whose polarization vector, $\hat{\varepsilon}$, defines the $z$ axis, $\hat{\varepsilon} \equiv \hat{z}$. The electric field and the vector potential are $\mathcal{F}$ $=\mathcal{F}_{0} \hat{\varepsilon} \cos \omega t$ and $\mathcal{A}=\mathcal{A}_{0} \hat{\varepsilon} \sin \omega t$, respectively, where $\omega$ is the frequency and $\mathcal{F}_{0}$ is the amplitude of the electric field, and where $\mathcal{A}_{0}=-c \mathcal{F}_{0} / \omega$.

In our model of a negative ion, the single active electron moves in the field of a Yukawa potential,

$$
W(r)=W_{0} \frac{e^{-\lambda r}}{r} .
$$

For both $\mathrm{H}^{-}$and $\mathrm{F}^{-}$, the parameter describing the range of the electron-atom interaction is taken to be $\lambda=1.0 \mathrm{a}$.u. The strength parameter is taken to be $W_{0}=-1.1$ a.u. for $\mathrm{H}^{-}$and $W_{0}=-5.0745$ a.u. for $\mathrm{F}^{-}$. With such a choice of parameters the initial (bound) energy of the valence $s$ electron in $\mathrm{H}^{-}$is $E_{0}=-0.0275654$ a.u. and that for the valence $p$ electron in $\mathrm{F}^{-}$is $E_{0}=-0.12498$ a.u., so that they practically coincide with the experimental values reported in Refs. [42,43]. For further details concerning these model potentials, see Refs. $[17,19,44,45]$. For a discussion of the sensitivity of ATD spectra to the particular choice of potential parameters, see the Appendix.

\section{B. Floquet approach}

The Hamiltonian, $H(t)$, of an effective one-electron ionic system interacting with a laser field described by the vector potential, $\mathcal{A}$, has the following form in the dipole velocity gauge:

$$
H(t)=H_{0}+\frac{1}{c} \mathcal{A}(t) \cdot \boldsymbol{p},
$$

where $H_{0}$ is the field-free Hamiltonian of the model ion,

$$
H_{0}=\frac{\boldsymbol{p}^{2}}{2}+W .
$$

In Eq. (3), $\boldsymbol{p}$ is the canonical momentum of the valence electron and $W$ is the effective electron-atom interaction defined in Eq. (1). Owing to the time periodicity of the Hamiltonian (2), we can represent the quasistationary state of the system by the so-called Floquet ansatz (for a review, see Ref. [46]),

$$
|\Phi(t)\rangle=e^{-i E t} \sum_{n} e^{-i n \omega t}\left|\phi_{n}^{(E)}\right\rangle
$$

where the index $n$ describes the net number of photons absorbed by the ion. The harmonic components $\left|\phi_{n}^{(E)}\right\rangle$ associated with the quasienergy $E$ are solutions of the infinite set of coupled equations that originate from the time-dependent Schrödinger equation, $i \partial_{t}|\Phi(t)\rangle=H(t)|\Phi(t)\rangle$, after substitution of Eqs. (2) and (4),

$$
\left(E+n \omega-U_{p}-H_{0}\right)\left|\phi_{n}^{(E)}\right\rangle=V_{+}\left|\phi_{n-1}^{(E)}\right\rangle+V_{-}\left|\phi_{n+1}^{(E)}\right\rangle,
$$

where $V_{+}$and $V_{-}$are the Fourier components of the dipole operator,

$$
V_{+} e^{-i \omega t}+V_{-} e^{i \omega t}=\frac{1}{c} \mathcal{A}(t) \cdot \boldsymbol{p} .
$$

The set of equations (5) constitute the eigenvalue problem for the quasienergy $E$ and the harmonic components $\left|\phi_{n}^{(E)}\right\rangle$, which must be solved with appropriate boundary conditions. Since the initially bound electron is detached by the intense laser field with absorption of $n$ laser photons, the harmonic components must be regular at the origin and represented at large distances by spherical waves,

$$
\left|\phi_{n}^{(E)}\right\rangle \sim \frac{e^{i k_{n} r}}{r}, \quad r \rightarrow \infty,
$$

where $k_{n}=\sqrt{2\left(E+n \omega-U_{p}\right)}$ is the wave number (with $U_{p}$ $=\mathcal{F}_{0}^{2} / 4 \omega^{2}$ being the ponderomotive energy). These boundary conditions result in a complex quasienergy $E$,

$$
E=E_{0}+\Delta-\frac{i}{2} \Gamma,
$$

where $E_{0}$ is the initial state energy of the active electron, $\Delta$ is its ac Stark shift, and $\Gamma$ is its total detachment rate (for more details, see, e.g., [18,44-49]). When the number of absorbed laser photons, $n$, is such that $\operatorname{Re} E+n \omega-U_{p}>0$, the $n$-photon 
detachment channel is open; otherwise, the detachment channel is closed. Thus, the minimum number of absorbed laser photons, $n_{0}$, necessary to detach an electron from the state with quasienergy $E$ may be defined by $n_{0}=1+[X]$, where $X$ $=\left(U_{p}-\operatorname{Re} E\right) / \omega>0$, unless $[X]=X$, in which case $n_{0}=X$. The infinite set of coupled equations (5), with boundary conditions (7), can only be solved by truncation at some point. One may then represent the harmonic components in terms of a discrete basis of complex radial Sturmian functions and spherical harmonics. Numerical results are obtained by solving the resulting eigenvalue problem for the quasienergies and the coefficients of the Sturmian expansion; for details of the numerical methods used, see Ref. [50]. Once the eigenvalue problem is solved, the photoelectron angular distributions can be calculated. Since the theoretical formulation is given in great detail elsewhere [44-48], we present here only the main formulas employed in our calculations. For a linearly polarized laser field, the differential detachment rate, $d \Gamma^{(n)} / d \Omega$, for $n$-photon detachment of photoelectrons with kinetic energy $E_{f}$ into the solid angle $d \Omega$ about the direction of the electron momentum $\boldsymbol{k}_{f}$ (where $E_{f}=\operatorname{Re} E+n \omega-U_{p}$ and $\left.k_{f}=\sqrt{2 E_{f}}\right)$ is defined by

$$
\frac{d \Gamma^{(n)}}{d \Omega}=2 \pi k_{f}\left|M_{n}\left(\boldsymbol{k}_{f}\right)\right|^{2},
$$

where $M_{n}\left(\boldsymbol{k}_{f}\right)$ is the $n$-photon detachment amplitude,

$$
M_{n}\left(\boldsymbol{k}_{f}\right)=\sum_{N} e^{i(N-n) \beta} J_{N-n}(-\alpha)\left\langle\boldsymbol{k}_{f}|W| \phi_{E}^{(N)}\right\rangle,
$$

where $\alpha$ and $\beta$ are real constants defined by

$$
\alpha \cos (\omega t-\beta)=\int^{t} d \tau \boldsymbol{k}_{f} \cdot \mathcal{A}(\tau) .
$$

Integration of the angular distribution (9) over all photoelectron momentum directions gives the partial rate, $\Gamma^{(n)}$, for $n$-photon detachment,

$$
\Gamma^{(n)}=\int \frac{d \Gamma^{(n)}}{d \Omega} d \hat{\boldsymbol{k}}_{f} .
$$

Since the sum of the partial rates equals the total detachment rate $\Gamma$, where $\Gamma=-2 \operatorname{Im} E$, the relation,

$$
\sum_{n \geqslant n_{0}} \Gamma^{(n)}=-2 \operatorname{Im} E
$$

provides a test of the numerical accuracy of our approach.

\section{Classical analysis}

Even though photodetachment and ionization are essentially quantum-mechanical phenomena, classical analyses have been successfully used to describe a number of features of ATI or ATD energy and angular distributions (see, e.g., Refs. [40,41,51-54]). For example, the experimental observation $[55,56]$ of pronounced side lobes in the angular distributions of highly energetic ATI electrons around $30^{\circ}$ and $45^{\circ}$ with respect to the laser-field polarization direction have been interpreted using a classical analysis [40]. As the com- parison of our quantum-mechanical results and the related classical predictions for highly energetic electrons is intended to be purely qualitative, we shall consider only those angular distributions that result from rescattered electrons. In principle, one should also take into account the contributions of high-energy detached electrons that do not rescatter from the atom. However, for the energies of interest (i.e., higher than $2 U_{p}$ ), the contributions of such electrons are negligible.

\section{Rescattering model}

Employing the classical model of Refs. [40,41], we consider here an electron that is detached at time $t_{0}$ (at the origin, with an initial velocity of zero) by a monochromatic, linearly polarized laser field. For times $t \geqslant t_{0}$, the electron is assumed to interact only with the laser field, which is described by the vector potential $\mathcal{A}=\mathcal{A}_{0} \hat{\varepsilon} \sin \omega t$. That is, we neglect the interaction of the electron with the short-range potential of the parent atom. The kinetic energy of the electron, $E_{f}$, averaged over the period of the laser field, $2 \pi / \omega$, and expressed in units of $2 U_{p}$, is (cf. Refs. [40,41])

$$
\begin{aligned}
\frac{E_{f}}{2 U_{p}}\left(\equiv \kappa^{2}\right)= & \sin ^{2} \omega t_{0}-2 \sin \omega t_{1}\left(\sin \omega t_{0}-\sin \omega t_{1}\right) \\
& \times\left(1 \pm \cos \theta_{0}\right),
\end{aligned}
$$

where $\theta_{0}$ is the angle (measured with respect to the negative $z$ axis) at which the electron rescatters from the atom at the time $t_{1}$, the time at which the electron returns to its parent atom for the first time,

$$
\cos \omega t_{1}-\cos \omega t_{0}+\omega\left(t_{1}-t_{0}\right) \sin \omega t_{0}=0 .
$$

In Eq. (14), the signs \pm correspond to the cases $\sin \omega t_{0}$ $\gtrless \sin \omega t_{1}$, which depend on the direction along the $z$ axis along which the electron is initially detached. The angle $\theta$ at which a rescattered electron with energy $E_{f}(14)$ is detected asymptotically (having been rescattered at the angle $\theta_{0}$ ) is given by the relation

$$
\sin \left(\theta-\theta_{0}\right)=\sin \theta \frac{\sin \omega t_{1}}{\left|\sin \omega t_{0}-\sin \omega t_{1}\right|},
$$

where $\theta$, as usual, is measured with respect to the $z$ axis.

The differential angular distribution for electrons of energy $E_{f}$ (14) that are ejected within a solid angle $d \Omega$ $\equiv 2 \pi \sin \theta d \theta$ following detachment by the laser field at time $t_{0}$ and rescattered at time $t_{1}$ by the parent atom is

$$
\frac{d P}{d \Omega}=\frac{\omega}{4 \pi^{2} \sin \theta}\left(\frac{d \theta}{d t_{0}}\right)^{-1},
$$

where the calculation of the derivative of the detection angle, $\theta$, with respect to the detachment time, $t_{0}$, requires use of Eqs. (14)-(16). In calculating this derivative, we assume that just before recolliding with its parent ion the electron moves along the negative $z$ direction, which implies the condition $\sin \omega t_{0}>\sin \omega t_{1}$. The result for the differential angular distribution in Eq. (17) is thus, 


$$
\begin{aligned}
\frac{d P}{d \Omega}= & \frac{\kappa \sin \omega t_{1}\left(\sin \omega t_{1}-\sin \omega t_{0}\right)}{4 \pi^{2} \cos \omega t_{0}}\left[\left(\sin \omega t_{0}-\sin \omega t_{1}\right)^{2}\right. \\
& \left.+\omega\left(t_{1}-t_{0}\right) \cos \omega t_{1}\left(\kappa \cos \theta+\sin \omega t_{0}\right)\right]^{-1}
\end{aligned}
$$

where

$$
\sin \omega t_{1}=\frac{\sin ^{2} \omega t_{0}-\kappa^{2}}{2\left(\kappa \cos \theta+\sin \omega t_{0}\right)},
$$

and with restrictions imposed on the values of $t_{0}$ and $\theta$ stemming from the fact that $\left|\sin \omega t_{1}\right| \leqslant 1$. The results in Eqs. (18) and (19) apply for detached electrons in the angular range $90^{\circ} \leqslant \theta \leqslant 180^{\circ}$. Results for $0^{\circ} \leqslant \theta \leqslant 90^{\circ}$ may be obtained by mirror reflection with respect to $\theta=90^{\circ}[40,41]$.

\section{Angle-dependence of the ATD plateau cutoff energy}

Here we derive the cutoff law for the high-energy ATD plateau electrons detected at an arbitrary angle $\theta$ in order to compare the classical predictions with our quantummechanical results. The maximum of the kinetic energy (14) determines the cutoff of the high-energy ATD plateau electrons that have been rescattered at the angle $\theta_{0}$ from the parent atom. The problem reduces to solving the equation $d E_{f} / d t_{0}=0$ subject to the condition (15). Introducing the new variables $\tau=\omega\left(t_{1}-t_{0}\right) / 2$ and $\sigma=\omega\left(t_{1}+t_{0}\right) / 2$, the result is

$$
\begin{gathered}
1+\left(1 \pm \cos \theta_{0}\right)[(\tau \cot \tau-1)(4 \tau \cot \tau-1) \\
\left.-\tau^{2}\left(3+\frac{\tau \cot \tau}{\tau \cot \tau-1}\right)\right]=0
\end{gathered}
$$

where $\sigma$ is given by

$$
\cot \sigma=\cot \tau-1 / \tau \text {. }
$$

Thus, for fixed rescattering angle, $\theta_{0}$, Eqs. (20) and (21) determine the variables $\tau$ and $\sigma$, which in turn determine $t_{1}$ and $t_{0}$. Equation (16) then gives the angle $\theta$ at which the electron is detected and Eq. (14) gives the cutoff energy at that angle.

Let us note that for backscattering (i.e., either $\theta_{0}=0^{\circ}$ and $\theta=0^{\circ}$ for $\sin \omega t_{0}>\sin \omega t_{1}$ or $\theta_{0}=180^{\circ}$ and $\theta=180^{\circ}$ for $\left.\sin \omega t_{0}<\sin \omega t_{1}\right)$, which is the most studied case in the literature, one obtains from (14) the well-known $10 U_{p}$ energy cutoff. An interesting question is whether the classical predictions for the energy cutoff of the ATD plateau agrees with results of quantum-mechanical calculations for intensities near multiphoton channel closings, since such channel closings are purely quantum phenomena. In the following section, we compare the classical predictions with the results of our quantum-mechanical numerical investigations for ATD energy and angular distributions at intensities in the vicinity of multiphoton channel closings, for two different ionic targets, $\mathrm{H}^{-}$and $\mathrm{F}^{-}$.

\section{RESULTS AND DISCUSSION}

In this section, we present our numerical results based on the Sturmian-Floquet theory described in Sec. II B. In Secs.
III A and III B we analyze the fixed-angle ATD spectra near channel closings: In Sec. III A we focus on the enhancements in the mid-plateau energy region, while in Sec. III B we discuss the direct detachment part of the ATD spectrum, which complements the non-CC analysis [57] for this region for electrons detached along the laser polarization axis. Angular distributions of detached electrons on the ATD plateau for intensities near the multiphoton CCs are presented in detail in Secs. III C and III D for plateau electrons in the mid- and high-energy plateau regions, respectively. Even though our focus is on the CC-induced enhancements of the electron angular distributions (cf. Sec. III C), we present also evidence for a new type of AD threshold behavior characteristic for highly energetic electrons (cf. Sec. III D).

Before presenting our results, we make two comments regarding the possibility of comparing our predictions with experiment. First, we note that although no focal averaging of our calculated angular distributions over the spatiotemporal intensity distribution of the electric field in the laser focus has been done, our predicted enhancements and AD structures are expected to survive such averaging. This has been shown to be the case for the AD structures in rare gas ATI spectra [58], and we fully expect this to be the case also for the ATD results we present here for $\mathrm{H}^{-}$and $\mathrm{F}^{-}$. Second, we note that owing to difficulties in the experimental determination of the laser intensity in the laser focus, flexibility is required in any comparison of theoretical results with experiment. For example, as a test of our codes and procedures we have compared our focal-averaged, fixed-angle ATD rates for $\mathrm{F}^{-}$with the experimental data of Kiyan and Helm [31] using the experimentally reported value of the peak intensity. These results (which are not shown, since they do not involve CC effects) agree qualitatively with those obtained in the experiment [31], but are considerably lower in magnitude, as was also found in other theoretical investigations by other methods $[35,36]$. Only when a significantly higher value of the peak intensity was employed did these other investigations $[35,36]$ obtain results that agreed better quantitatively with the experimental results.

In what follows results are presented in all cases for a linearly polarized laser field having frequency $\omega$ $=0.0043$ a.u. (wavelength $\lambda=10.6 \mu \mathrm{m}$ ) in the case of $\mathrm{H}^{-}$and frequency $\omega=0.0253 \mathrm{a} . \mathrm{u}$. (wavelength $\lambda=1.8 \mu \mathrm{m}$ ) in the case of $\mathrm{F}^{-}$.

\section{A. ATD enhancement vs detachment angle}

In Figs. 1 and 2 we present fixed-angle ATD electron spectra for the hydrogen and fluorine negative ions at laser intensities below, above, and at the intensity at which the 10and nine-photon detachment channels become closed for $\mathrm{H}^{-}$ and $\mathrm{F}^{-}$, respectively. In general, the enhancement of the high-energy ATD plateau manifests itself most strongly at the closing of even or odd multiphoton channels for target atoms or ions having valence $s$ or $p$ electrons, respectively $[15-17,20]$. This is why we exhibit results for the 10-photon channel closing for $\mathrm{H}^{-}$and the nine-photon channel closing for $\mathrm{F}^{-}$. These channel closings occur for intensities of approximately $3.99 \times 10^{10} \mathrm{~W} / \mathrm{cm}^{2}$ for $\mathrm{H}^{-}$and 9.3 


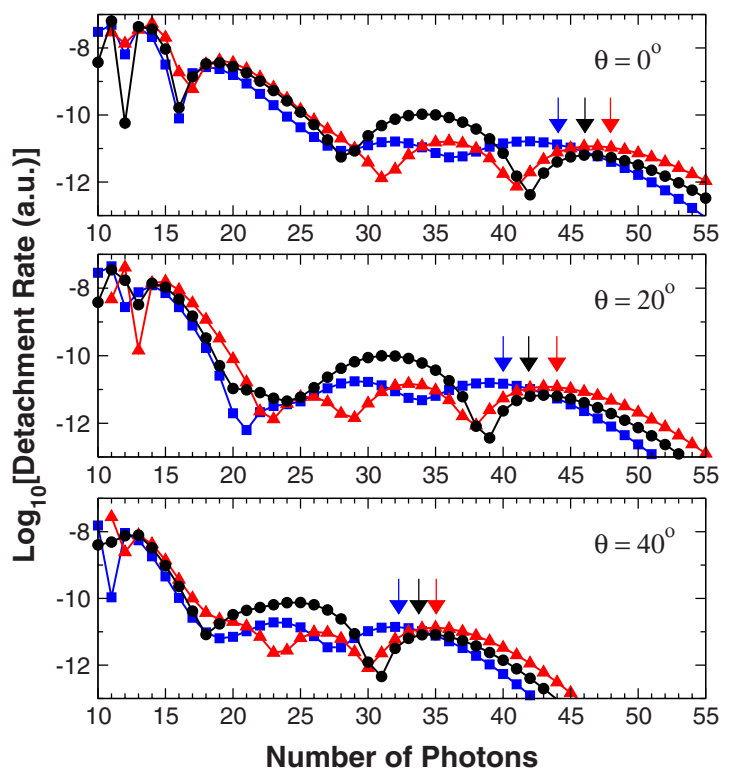

FIG. 1. (Color online) Fixed-angle ATD spectra for $\mathrm{H}^{-}$detachment by a linearly polarized laser field of frequency $\omega$ $=0.0043$ a.u. for three laser field intensities in the vicinity of the closure of the 10-photon detachment threshold. Results for intensities $I=3.79,3.99$, and $4.19 \times 10^{10} \mathrm{~W} / \mathrm{cm}^{2}$ are denoted by blue squares, black circles, and red triangles, respectively, and are connected to guide the eye. The three arrows of the corresponding color each mark the classical energy cutoff, calculated as discussed in Sec. II C 2.

$\times 10^{12} \mathrm{~W} / \mathrm{cm}^{2}$ for $\mathrm{F}^{-}$. At these intensities, pronounced enhancements (up to an order of magnitude) of groups of ATD peaks can be observed in Figs. 1 and 2. For $\mathrm{H}^{-}$(respectively, $\mathrm{F}^{-}$), these regions of enhanced detachment rates range approximately from $n=30-39$ (respectively, $n=34-42$ ) for $\theta$ $=0^{\circ}$, from $n=27-36$ (respectively, $n=31-38$ ) for $\theta=20^{\circ}$, and from $n=20-29$ (respectively $n=22-29$ ) for $\theta=40^{\circ}$, where $n$ is the number of photons absorbed by the detached electron and $\theta$ is the detachment angle. The enhancement of the ATD rates clearly occurs in the low- and mid-energy part of the rescattering plateau. (The three colored arrows in each panel mark the classically predicted high-energy cutoff for each of the three plateaus; cf. Sec. II C 2.)

What is interesting in the results shown in Figs. 1 and 2 is that the enhancements are of comparable magnitude both on axis (i.e., $\theta=0^{\circ}$ ) and off axis (i.e., $\theta=20^{\circ}, 40^{\circ}$ ), although the higher the detachment angle the lower the energy range of the enhancement. This shift of the enhancement to lower energies with increasing detachment angle is consistent with the fact that rescattering, which gives rise to the ATD plateau, occurs predominantly for electrons having small detachment angles and vanishes for large angles (see, e.g., Refs. [54,57]). Comparing the different panels in each figure, one may also conclude that plateau enhancements at lower energies for large detachment angles will be obscured by direct electron detachment at smaller angles in the angleintegrated detachment rates. As presented in Figs. 1 and 2, this remark refers specifically to the plateau enhancement in the ATD spectrum for $\theta=40^{\circ}$, and only marginally to that for $\theta=20^{\circ}$.

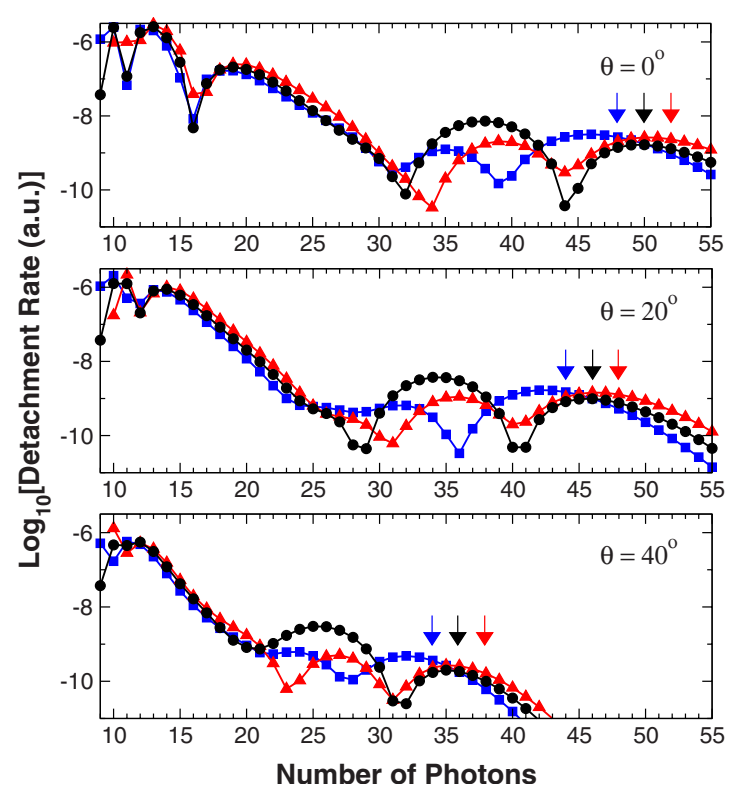

FIG. 2. (Color online) Fixed-angle ATD spectra for $\mathrm{F}^{-}$detachment by a linearly polarized laser field of frequency $\omega$ $=0.0253$ a.u. for three laser field intensities in the vicinity of the closure of the nine-photon detachment threshold: $I=8.9$ (blue squares), 9.3 (black circles), and 9.7 (red triangles) $\left(\times 10^{12}\right) \mathrm{W} / \mathrm{cm}^{2}$. The rates have been averaged over the projections of the initial angular momentum, $\ell=1$. The arrows are defined as in Fig. 1.

We note finally that very recently an investigation of ATI for $\mathrm{He}, \mathrm{Ar}, \mathrm{Kr}$, and $\mathrm{Xe}$ plateau electrons in the vicinity of channel closings has appeared [58]. In that work, an improved strong field approximation has been employed to calculate the ATI energy spectrum for $\mathrm{Kr}$ for various fixed electron ejection angles and for different laser intensities, including those at low-energy channel closings. A main finding for $\mathrm{Kr}$ is that the plateau enhancements are found to appear at somewhat lower energies as the electron ejection angle (measured from the laser polarization axis) increases [58]. This result is consistent with our findings for $\mathrm{H}^{-}$and $\mathrm{F}^{-}$ shown in Figs. 1 and 2.

\section{B. Direct detachment near channel closings}

In Fig. 3 we present fixed-angle ATD rates for both $\mathrm{H}^{-}$ and $\mathrm{F}^{-}$by a laser field having an intensity corresponding to closure of the 10-photon detachment channel in the case of $\mathrm{H}^{-}$and of the nine-photon detachment channel in the case of $\mathrm{F}^{-}$. (See the figure caption for the laser parameters used.) The ATD rates are plotted versus electron energy for three different detachment angles. Two different sets of results are compared: Our essentially exact Sturmian-Floquet theory results, which include interaction of the active electron with the atomic core, and Keldysh theory results [obtained using Eq. (33) of Ref. [38]], which ignore interactions of the active electron with the atomic core. One observes that for each ejection angle and each ionic target, very good agreement of our numerical results with predictions based on the Keldysh approximation is found in the low-energy parts of the spec- 


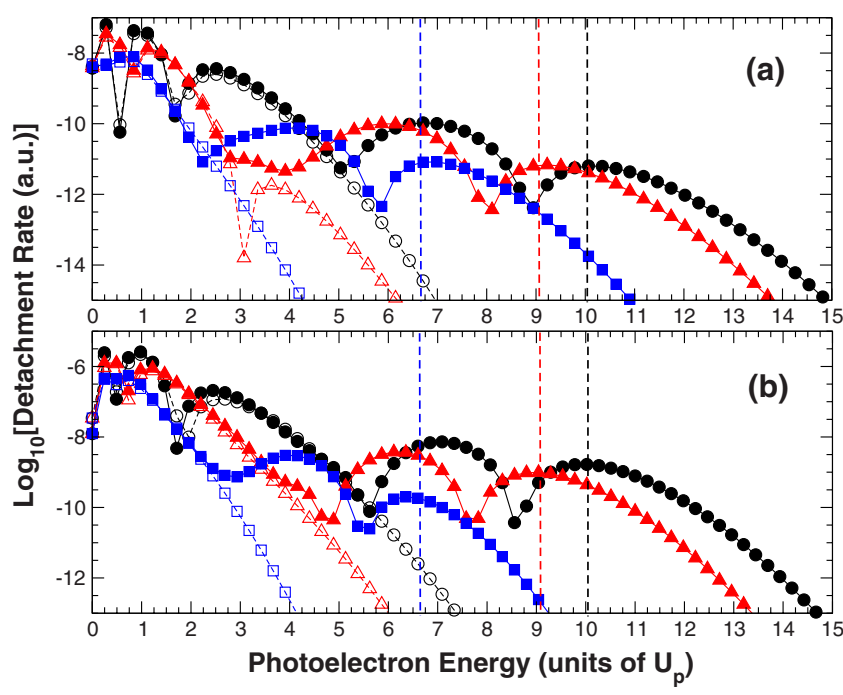

FIG. 3. (Color online) Comparison of the present SturmianFloquet detachment rates (filled symbols) with results based on Keldysh theory [38] (open symbols) as a function of the detached electron energy in units of the ponderomotive potential energy, $U_{p}$. Panel (a) shows ATD spectra for $\mathrm{H}^{-}$for a linearly polarized laser field of frequency $\omega=0.0043$ a.u. and intensity $I=3.99$ $\times 10^{10} \mathrm{~W} / \mathrm{cm}^{2}$ just before closure of the ponderomotively shifted 10-photon detachment channel. Panel (b) shows ATD spectra for $\mathrm{F}^{-}$ for a linearly polarized laser field of frequency $\omega=0.0253$ a.u. and intensity $I=9.3 \times 10^{12} \mathrm{~W} / \mathrm{cm}^{2}$ just before closure of the ninephoton detachment threshold. In each panel, different curves correspond to different ejection angles of the detached electron: $\theta=0^{\circ}$ (filled and open black circles), $20^{\circ}$ (filled and open red triangles), and $40^{\circ}$ (filled and open blue squares). The vertical dashed lines indicate the classical ATD high energy plateau cutoffs, calculated as discussed in Sec. II C 2.

tra. This agreement is expected since, in the energy region below the ATD plateau, rescattering effects that are ignored in Keldysh-type treatments are not significant.

As the angle with respect to the laser polarization axis increases, we observe that all structures in the ATD spectrum move to lower detached electron energies. As shown in Fig. 3 , this is true of the energy location of the last local maximum before the onset of the ATD plateau, it is true of the onset of the ATD plateau, and it is true of the high-energy cutoff of the ATD plateau. This finding is consistent with similar findings by others (e.g., see Refs. [54,57]) for laser intensities not corresponding to the closing of a multiphoton detachment channel.

An interesting feature of Fig. 3 is that for electrons ejected along the polarization axis ( $\mathrm{cf}$. the curves for $\theta=0^{\circ}$ ), the directly detached electrons for both $\mathrm{H}^{-}$and $\mathrm{F}^{-}$have a local maximum at approximately $2.5 U_{p}$ and then decrease rapidly until the onset of the plateau (at which point our Sturmian-Floquet results oscillate with increasing energy, thereby forming the ATD plateau, while the Keldysh approximation results continue to decrease sharply). This finding of a common energy for the last local maximum in the spectrum of directly detached electrons is a coincidence for the particular laser parameters used. In Fig. 4 it is shown that for directly detached electrons ejected along the polarization

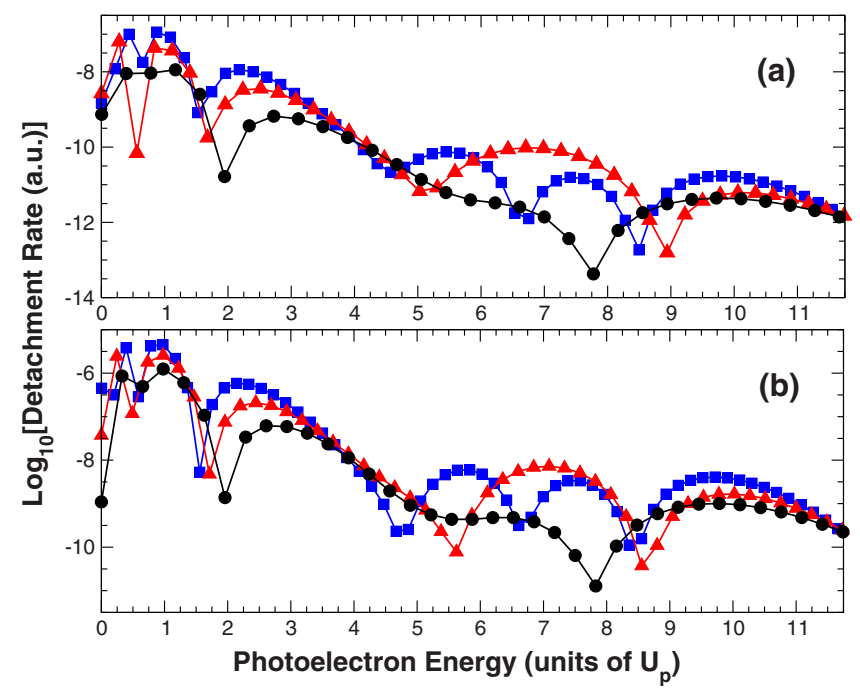

FIG. 4. (Color online) ATD spectra for detached electrons detected along the direction of linear laser polarization for intensities corresponding to the closure of multiphoton detachment channels. (a) ATD for $\mathrm{H}^{-}$for a laser field of frequency $\omega=0.0043$ a.u. and intensity $I=2.87,3.99$, and $5.12 \times 10^{10} \mathrm{~W} / \mathrm{cm}^{2}$, corresponding, respectively, to closure of the nine-, 10-, and 11-photon detachment channels (black dots, blue triangles, and blue squares, respectively); (b) ATD for $\mathrm{F}^{-}$for a laser field of frequency $\omega=0.0253$ a.u. and intensity $I=6.97,9.3$, and $11.72 \times 10^{12} \mathrm{~W} / \mathrm{cm}^{2}$, corresponding, respectively, to closure of the eight-, nine-, and 10-photon detachment channels (black dots, blue triangles, and blue squares, respectively). The rates in panel (b) have been averaged over the projections of the initial angular momentum, $\ell=1$.

axis, the last local maximum before the onset of the ATD plateau shifts toward lower energies as the laser intensity increases. For intensities corresponding to the closures of the nine-, 10-, and 11-photon detachment channels of $\mathrm{H}^{-}$and to the closures of the eight-, nine-, and 10-photon detachment channels of $\mathrm{F}^{-}$, the energy location of this local maximum shifts from somewhat less than $3 U_{p}$ to somewhat more than $2 U_{p}$ in both cases. These results apparently disagree with the conclusions of other researchers regarding their results for different laser parameters (cf. Fig. 2 of Ref. [31] and Sec. III B of Ref. [57]). Specifically, the results of Ref. [57] indicate that the last local maximum in the detachment rates for $\mathrm{H}^{-}$and $\mathrm{F}^{-}$occur at electron energies of $2 U_{p}$ and $3 U_{p}$, respectively, for detachment along $\theta=0^{\circ}$. This difference is attributed by the authors of Ref. [57] to an angularmomentum-sensitive interference of two different contributions to the detachment amplitude in each case. A similar argument was presented earlier in Ref. [31] based on their Eq. (1), which is derived from Eq. (33) of Ref. [38] for the special case when the kinetic energy, $E_{f}$, of the active electron satisfies $E_{f} \gg\left|E_{0}\right|$ and $E_{f} \gg U_{p}$ (i.e., is much larger than the initial binding energy, $-E_{0}$, of the active electron as well as the ponderomotive potential energy). The authors of Ref. [57] note that their results agree with the theoretical results of Ref. [31]. In contrast to these predictions of an initial state symmetry dependence of the energy location of the last local maximum before the onset of the plateau, our results in Figs. 3 and 4 show that, regardless of the initial state symmetry, 

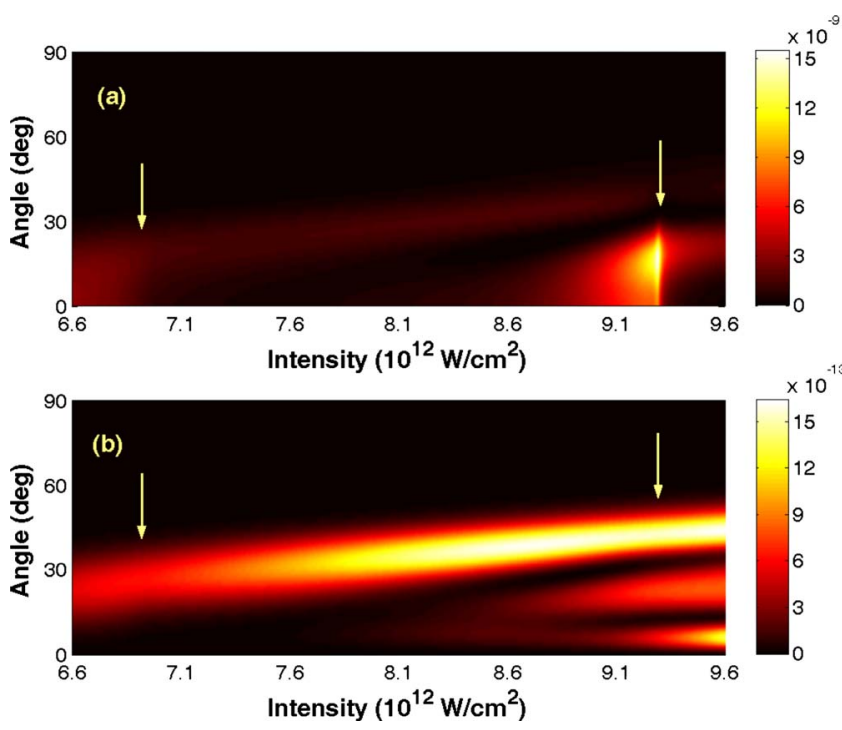

FIG. 5. (Color online) ATD angular distributions for $\mathrm{F}^{-}$detachment by 34 linearly polarized $1.8 \mu \mathrm{m}$ laser photons over a range of laser intensities that includes the closure of the eight- and ninephoton detachment thresholds (at $6.97 \times 10^{12} \mathrm{~W} / \mathrm{cm}^{2}$ and 9.3 $\times 10^{12} \mathrm{~W} / \mathrm{cm}^{2}$, respectively, as marked by arrows). Panels (a) and (b) correspond to results for electron orbital angular-momentum projection quantum numbers $m=0$ and $m= \pm 1$, respectively. The scale on the right-hand side of each panel indicates the magnitudes (in a.u.) of the angular distributions.

the energy location of this maximum shifts to lower energies with increasing laser intensities corresponding to similar multiphoton channel closings in both $\mathrm{H}^{-}$and $\mathrm{F}^{-}$.

A possible resolution of the difference between our results and those presented in Fig. 2 of Ref. [31] and in Figs. 3, 4 and 5 of Ref. [57] may be that the conditions upon which Eq. (1) of Ref. [31] was derived do not apply in our case. In our results shown in Fig. 3, for example, the laser intensities are such that the 10-photon detachment channel in the case of $\mathrm{H}^{-}$ and the nine-photon detachment channel in the case of $\mathrm{F}^{-}$are just becoming closed. Close to the $m$-photon detachment threshold, a detached electron that has absorbed $n$ laser photons $(n>m)$ has kinetic energy $E_{f}=(n-m) \omega$. In order for this electron to have a kinetic energy much greater than its effective binding energy, one must therefore have $n \gg 2 m$. For the intensities in our results, therefore, this would mean $n \gg 20$ for $\mathrm{H}^{-}$and $n \gg 18$ for $\mathrm{F}^{-}$. As shown in our Figs. 1 and 2, the energies at which such conditions are satisfied are well beyond the region of direct detachment. It appears therefore that the analysis developed in $[31,57]$ cannot be rigorously applied to a description of the low-energy ATD spectra for intensities near the multiphoton detachment channel closings considered in this work.

\section{Electron angular distributions in the low- to intermediate- energy region of the ATD plateau}

In Fig. 5, we present color mappings of the angular distributions for electrons detached from $\mathrm{F}^{-}$by absorption of $n=34$ linearly polarized photons (of frequency 0.0253 a.u.) as a function of laser intensity. The laser intensities span the region over which the ponderomotive potential closes the eight- and nine-photon detachment channels (indicated by the arrows). Owing to the axial symmetry of the problem, the angular distributions are only shown for angles in the range of $0-90^{\circ}$. The panels (a) and (b) correspond, respectively, to initial state electron magnetic quantum numbers, $m=0$ and $m= \pm 1$. (Note that the magnitudes of the $m= \pm 1$ results are three orders of magnitude smaller than those for $m=0$, as also shown later.) Whereas one observes a pronounced increase of the detachment rate for $m=0$ at the nine-photon CC over a very narrow range of laser field intensities, only a very subtle, barely visible change in the rate may be seen at the eight-photon CC. This is an illustration of a general law that governs the dynamics of detachment at the thresholds of multiphoton transitions. At such thresholds, the Wigner threshold law [21] predicts that the energy dependence of the partial detachment rates depends strongly on the initial state symmetry (i.e., on the orbital angular momentum of the valence electron) and on the number of absorbed photons. As has been described in Refs. [15-17,19,20], for a linearly polarized laser field, this leads to the enhancement of the rates for electron detachment on the ATD plateau whenever the laser intensity leads to the closing of an even- or oddmultiphoton detachment channel for an $s$ (as in $\mathrm{H}^{-}$) or $p$ (as in $\mathrm{F}^{-}$) valence electron, respectively. The angular distributions follow the same threshold law as the partial rates do. Because the behaviors of the ADs for $\mathrm{H}^{-}$in the vicinity of even- and odd-multiphoton CCs are similar to those for the $m=0$ valence state of $\mathrm{F}^{-}$in the vicinity of odd- and evenmultiphoton $\mathrm{CCs}$, respectively, we do not present here the corresponding color mappings of the angular distributions for $\mathrm{H}^{-}$detachment.

The large increase in the magnitudes of the angular distributions for electrons on the ATD plateau at laser intensities corresponding to the closure of a particular multiphoton detachment channel is shown more quantitatively in Fig. 6. In this figure, we compare the ADs for an $s$ electron $\left(\mathrm{H}^{-}\right)$[panels (a) and (b)] and a $p$ electron $\left(\mathrm{F}^{-}\right)$[panels (c) and (d)]. In both cases the electron kinetic energy is the same: Either $6 U_{p}$ [panels (a) and (c)] or $7 U_{p}$ [panels (b) and (d)]. For $\mathrm{F}^{-}$, the rates shown are averaged over the projections of the initial orbital angular momentum, $\ell=1$. As specified in the caption to Fig. 6, the four laser intensities for which results are shown span the closure of the nine- and 10-photon $\left(\mathrm{H}^{-}\right)$and the eight- and nine-photon $\left(\mathrm{F}^{-}\right)$detachment channels. What is most striking in Fig. 6 is that at the intensities that close the 10-photon detachment channel in the case of $\mathrm{H}^{-}$and the nine-photon detachment channel in the case of $\mathrm{F}^{-}$, the angular distributions for ATD plateau electrons (represented by the solid black curves) exhibit very large maxima in a finite angular interval. These maxima are about an order of magnitude larger than those in the ADs corresponding to intensities higher or lower than that for the solid black curve in each panel. We thus see that the ATD plateau enhancements at multiphoton CCs are not only confined to the lower energy portion of the ATD plateau, but also that for particular energies in this region the ATD electron ADs are enhanced only within a finite angular range. At higher angles there is almost no significant increase at laser intensities corresponding to multiphoton CCs. On the other hand, at the closing of the 


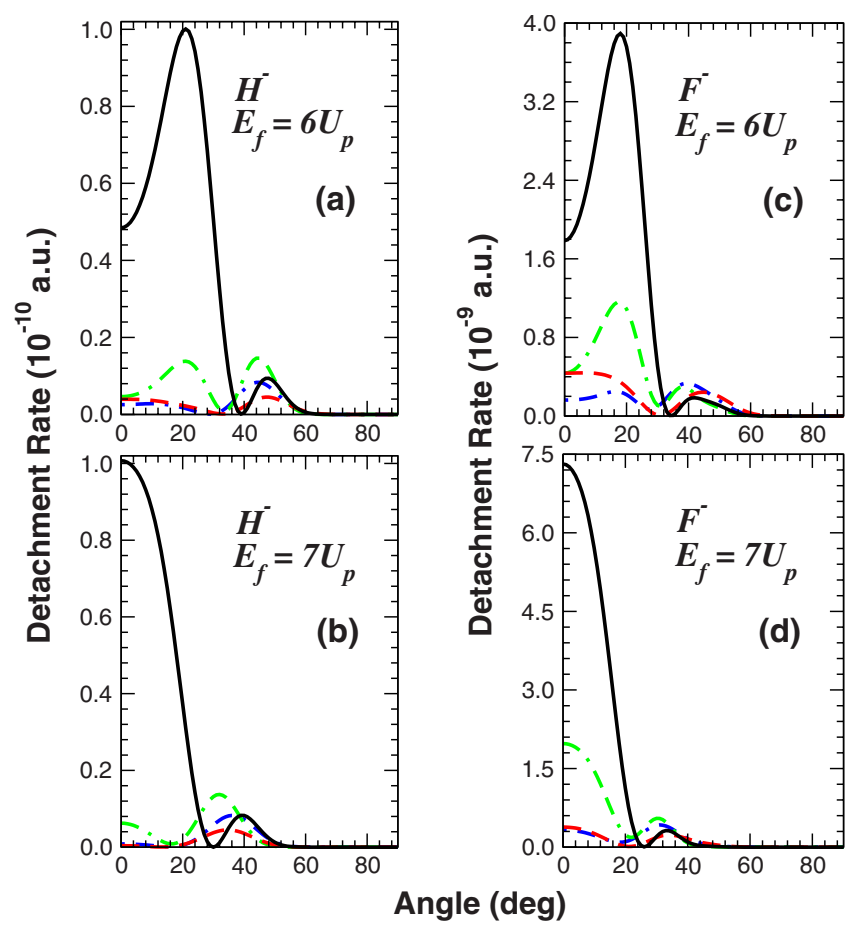

FIG. 6. (Color online) Intensity dependence of electron angular distributions on the ATD plateau for electron kinetic energies of $6 U_{p}$ and $7 U_{p}$. Results are shown for both $\mathrm{H}^{-}$and $\mathrm{F}^{-}$ATD by linearly polarized laser fields having frequencies $\omega=0.0043$ a.u. and $\omega=0.0253$ a.u., respectively. In each panel, results are presented for four values of intensity, $I$, that span the closing of the nine- and 10-photon detachment channels in the case of $\mathrm{H}^{-}$and the eight- and nine-photon detachment channels in the case of $\mathrm{F}^{-}$. (a) $\mathrm{H}^{-}$detachment with intensity in units of $10^{10} \mathrm{~W} / \mathrm{cm}^{2}, I=2.87$ (dashed red curve, $n=24$, nine-photon channel closing), $I=3.3$ (dashed-dotted blue curve, $n=27$ ), $I=3.99$ (solid black curve, $n=31$, 10-photon channel closing), and $I=4.24$ (dashed-dashed-dotted green curve, $n=33$ ), where $n$ is the number of photons absorbed by electrons having kinetic energy $6 U_{p}$; (b) same as in panel (a) except that the electron kinetic energy of $7 U_{p}$ involves absorption of $n=28,30,35$, and 39 photons, respectively; (c) $\mathrm{F}^{-}$detachment with intensity in units of $10^{12} \mathrm{~W} / \mathrm{cm}^{2}, I=6.97$ (dashed red curve, $n=26$, eightphoton channel closing), $I=8.1$ (dashed-dotted blue curve, $n=30$ ), $I=9.3$ (solid black curve, $n=34$, nine-photon channel closing), and $I=9.8$ (dashed-dashed-dotted green curve, $n=35$ ), where $n$ is the number of photons absorbed by electrons having kinetic energy $6 U_{p}$; (d) same as in panel (c) except that the electron kinetic energy of $7 U_{p}$ involves absorption of $n=29,33,38$, and 40 photons, respectively. The rates for $\mathrm{F}^{-}$have been averaged over the projections of the initial orbital angular momentum, $\ell=1$.

nine-photon channel in the case of $\mathrm{H}^{-}$and the eight-photon detachment channel in the case of $\mathrm{F}^{-}$, the angular distributions for ATD plateau electrons (represented by the dashed red curves) do not exhibit any enhancement. This illustrates the symmetry dependence of the ATD enhancements, stemming from the Wigner threshold law [21].

The variation of $\mathrm{F}^{-}$ATD plateau enhancements with detached electron kinetic energy, electron emission angle, and laser intensity is shown in Fig. 7. The results in this figure are similar to the results for $n=34$ shown in Fig. 5, but are presented for a much narrower range of laser intensities in the region of the closure of the nine-photon detachment channel (at approximately $9.3 \times 10^{12} \mathrm{~W} / \mathrm{cm}^{2}$ ). Each panel corresponds to a different ATD plateau peak (varying from $n=27-41$ ). Since the $m= \pm 1$ ADs are smaller (by three orders of magnitude) than the corresponding $m=0$ ADs and since, as shown in Fig. 5, the $m= \pm 1$ ADs show no evident enhancements in the region of intensity at which the ninephoton channel closing occurs, only the $m=0$ data are presented. The most interesting feature of the ADs presented in Fig. 7 is the abrupt increase in magnitude over a finite angular interval in the $\mathrm{AD}$ beginning at laser intensities just before the nine-photon CC. As we have already discussed with regard to the results in Fig. 2, but which is illustrated better in Fig. 7, the enhancement of high-order $\mathrm{ADs}$ is not restricted to only those electrons that move along the laser field polarization direction. For lower ATD peaks, $n$, the enhancement is observed far from the polarization axis, in the region of $\theta=30^{\circ}$. However, with increasing $n$ the angular interval over which enhancement occurs shifts toward lower angles, i.e., closer to the polarization axis. To reiterate, at the low energy end of the ATD plateau, evidence of CC enhancements of the ATD rates appears first at large angles and only at higher energies does it appear along the laser polarization axis at $\theta=0^{\circ}$.

The variations of the detached electron ADs with changes in laser intensity in the vicinity of multiphoton CCs is examined in more quantitative detail for $\mathrm{F}^{-}$in Figs. 8-10. In Fig. 8 we present the ADs for electrons for three laser intensities that are below, at, and above the intensity corresponding to the closing of the nine-photon detachment threshold. The three panels in this figure show the ADs for detached electrons that have absorbed $n=30,34$, and 38 laser photons, all of which lie in the low- to intermediate-energy region of the ATD plateau (cf. Fig. 2). The respective rates have been averaged over the projections of the initial orbital angular momentum of the outer electron, $\ell=1$. One clearly sees in this figure that the pronounced enhancement of one of the maxima in the ADs corresponding to the multiphoton $\mathrm{CC}$ intensity (cf. the solid black curves) shifts from significantly off axis to smaller angles with increasing electron kinetic energy. For $n=30$ the enhanced maximum is located about the angle $\theta=30^{\circ}$, while for $n=34$ the enhanced maximum peaks at approximately $\theta=17^{\circ}$, and for $n=38$ the peak is at $\theta=0^{\circ}$. Note that in Fig. 8 the changes in laser intensity span a range of only about $6.5 \%$. Nevertheless these small changes produce large enhancements of the detached electron ADs.

For comparison, we show in Fig. 9 the ADs for detached electrons located below $\left(n=15, E_{f}<2 U_{p}\right)$ and above $(n=55$, $\left.E_{f}>10 U_{p}\right)$ the ATD plateau for the same laser parameters as in Fig. 8. In contrast to the nonmonotonic behavior of the plateau electron ADs with variations of intensity in the vicinity of the nine-photon CC (cf. Fig. 8), one observes in Fig. 9 that the ADs for electrons both below and above the ATD plateau change monotonically with increasing laser field intensity.

It should be emphasized that the large enhancements in the ADs for ATD plateau electrons shown for $\mathrm{F}^{-}$in Fig. 8 occur only for intensities in the vicinity of odd multiphoton channel closures owing to the $p$-state symmetry of the va- 


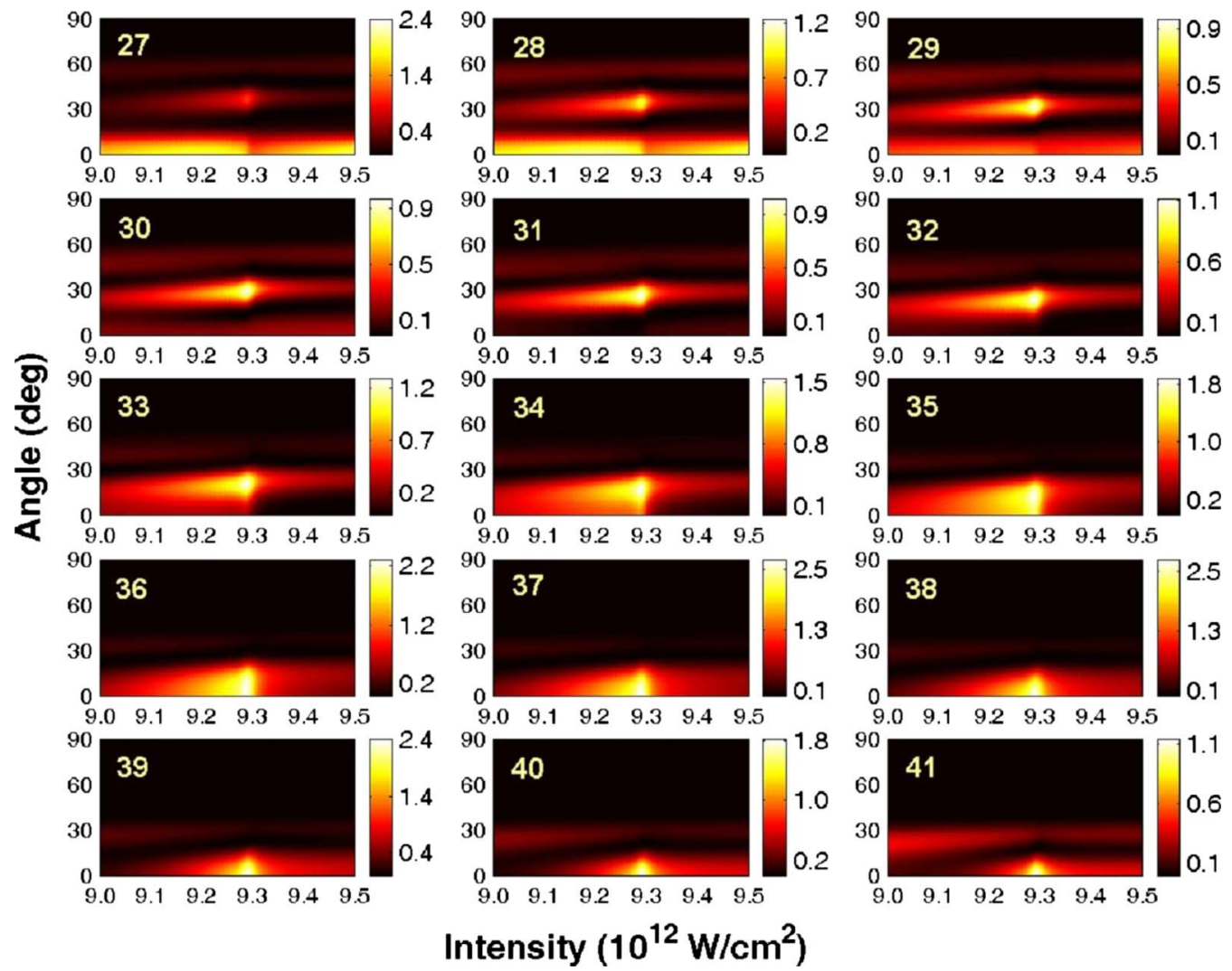

FIG. 7. (Color online) Intensity dependence of electron angular distributions on the ATD plateau for $\mathrm{F}^{-}$detachment by $n$ photons (where $n$ is indicated in each panel) having linear polarization and frequency $\omega=0.0253$ a.u. The laser intensities span the region over which the nine-photon detachment channel becomes closed. Results are shown only for electrons having orbital angular-momentum projection, $m$ $=0$, as those for $m= \pm 1$ are negligible and show no evident enhancement at the CC. The scale on the right-hand side of each panel indicates the magnitudes of the detachment rates (given in units of $10^{-8}$ a.u.).

lence electron. (For $\mathrm{H}^{-}$, owing to the $s$-state symmetry of the valence electron, the enhancements in the ADs for ATD plateau electrons occur only for intensities in the vicinity of even multiphoton channel closures.) We demonstrate this initial state symmetry rule in Fig. 10, which shows the variation of the $\mathrm{ADs}$ for $\mathrm{F}^{-}$detachment for laser intensities in the vicinity of the eight-photon CC. In contrast to the oddchannel closing results shown in Fig. 8, for laser intensities at the eight-photon (even) channel closing, the detached electron ADs along the ATD plateau (i.e., for $n=30,33$, and 36) show almost monotonic changes with increases in laser intensity. In particular, there are no dramatic changes in either the shapes or in the magnitudes of the ADs.

\section{Angular distributions near the high-energy cutoff of the ATD plateau}

Although ATI (ATD) plateau enhancements of the ionization (detachment) rates integrated over all ionized (detached) electron angles have only been predicted or observed for electron energies in the low- to intermediate-energy region of the ATI (ATD) plateau, we show here that the ADs for plateau electrons having energies near the plateau cutoffs show characteristic changes in shape with changes in laser intensity in the vicinity of multiphoton CCs. Also, these changes in shape of the ADs have a quantum-mechanical origin, as

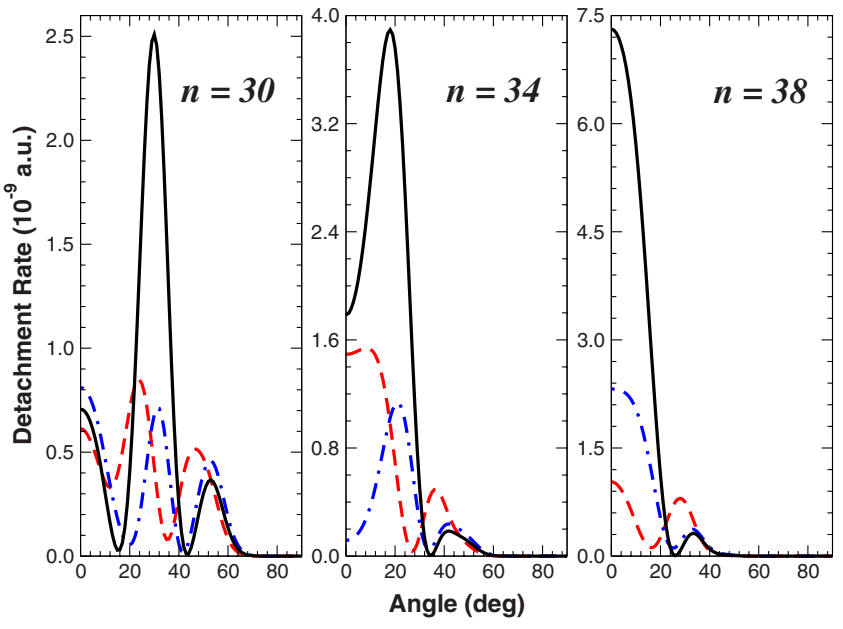

FIG. 8. (Color online) Variation with intensity of detached electron angular distributions for ATD of $\mathrm{F}^{-}$by $n$ linearly polarized laser photons of frequency $\omega=0.0253$ a.u., where $n=30,34$, and 38 . In each panel, results are given for three laser field intensities (in units of $10^{12} \mathrm{~W} / \mathrm{cm}^{2}$ ) that lie, respectively, just below, at, and just above the intensity corresponding to the closure of the nine-photon detachment channel: $I=8.9$ (dashed red curves); $I=9.3$ (solid black curves); and $I=9.5$ (dashed-dotted blue curves). Results are averaged over the projections of the initial orbital angular momentum, $\ell=1$. 


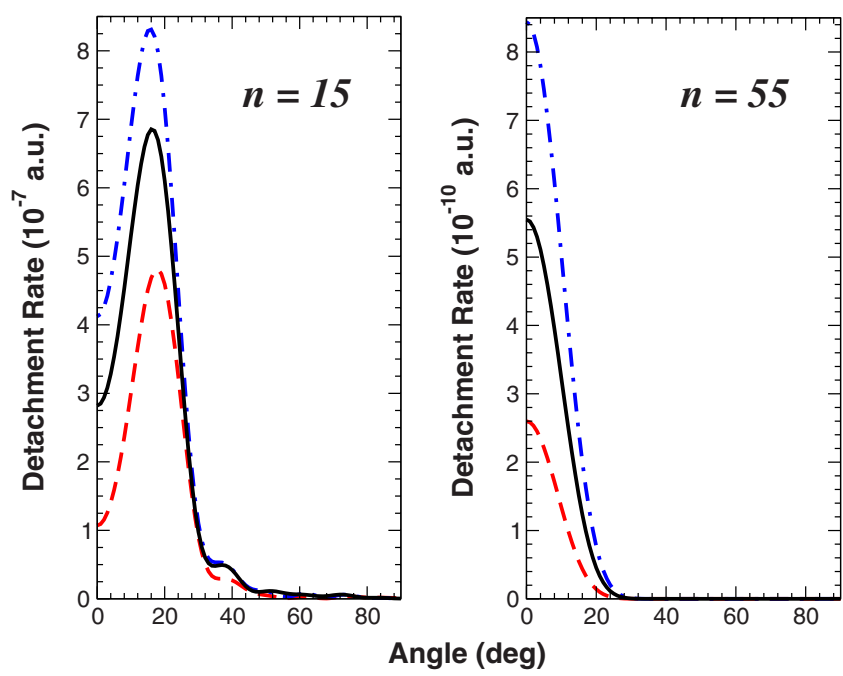

FIG. 9. (Color online) Same as in Fig. 8 but for ATD of $\mathrm{F}^{-}$by, respectively, 15 and 55 photons.

comparison with classical predictions for the ADs shows. A classical analysis has been successfully applied to the description of the related ATI angular distributions having pronounced off-axis maxima at angles between $30^{\circ}$ and $45^{\circ}$ with respect to the laser field direction [40]. This behavior of the ADs has been associated with the ATI peaks in the energy region around $8-9 U_{p}$. (For the corresponding experimental works, see Refs. $[53,55]$.) In this energy region, which corresponds to the end of the plateau, the ATI (ATD) energy spectra no longer exhibit intensity-sensitive enhancements. In Fig. 11 we present a comparison of the classical ADs (right-hand panel), calculated as described in Sec. II C 1, with the results of our quantum-mechanical investigations of $\mathrm{H}^{-}$detachment (left-hand panel). In both cases, the detached electron has a kinetic energy $E_{f}=8 U_{p}$. Different curves in the left-hand panel correspond to different laser intensities and to different numbers of absorbed laser photons.

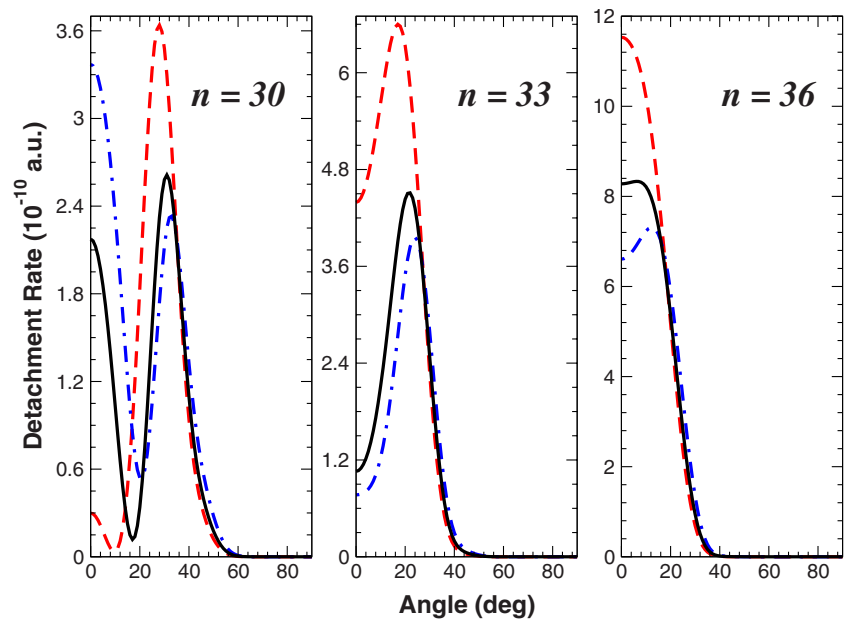

FIG. 10. (Color online) Same as in Fig. 8 but for ATD of $\mathrm{F}^{-}$by 30,33 , and 36 photons, and for three different laser field intensities (in units of $10^{12} \mathrm{~W} / \mathrm{cm}^{2}$ ) that lie, respectively, just below, at, and just above the intensity corresponding to the closure of the eightphoton detachment channel: $I=6.8$ (dashed red curves); $I=6.97$ (solid black curves); and $I=7.2$ (dashed-dotted blue curves).

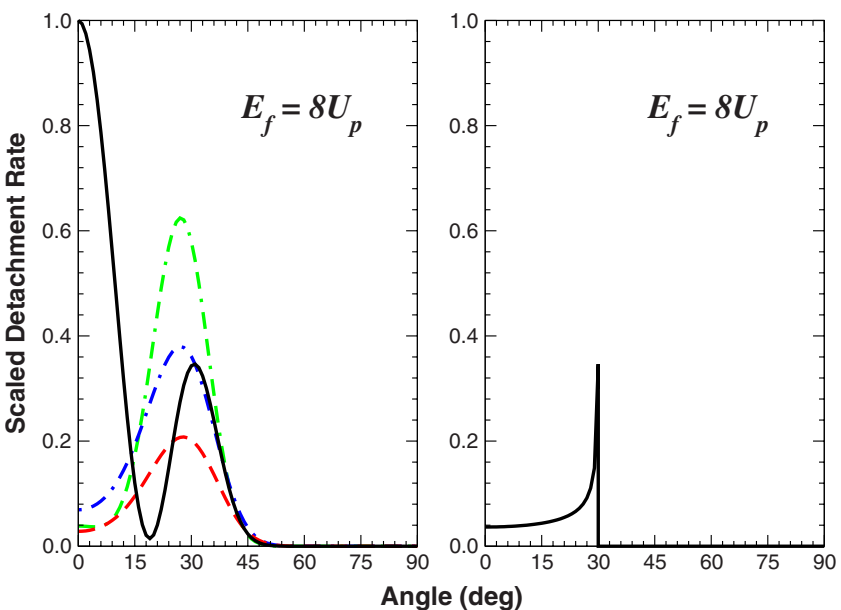

FIG. 11. (Color online) Comparison of quantum-mechanical (left-hand panel, present results) and classical (right-hand panel, cf. Sec. II C 1) predictions for the angular distributions of electrons detached from $\mathrm{H}^{-}$with kinetic energy $E_{f}=8 U_{p}$ by a linearly polarized laser field of frequency $\omega=0.0043$ a.u. The quantum results are presented for four different laser intensities (in units of $10^{10} \mathrm{~W} / \mathrm{cm}^{2}$ ) that span the closing of the nine- and 10-photon detachment channels: $I=2.87$ (dashed red curve, $n=30$, nine-photon CC), $I=3.3$ (dashed-dotted blue curve, $n=33$ ), $I=3.99$ (solid black curve, $n=39,10$-photon CC), and $I=4.24$ (dashed-dashed-dotted green curve, $n=41$ ), where $n$ is the number of photons absorbed by electrons having kinetic energy $8 U_{p}$. All results are relative, with the quantum results for $I=3.99 \times 10^{10} \mathrm{~W} / \mathrm{cm}^{2}$ (solid black curve) set equal to unity at the angle $0^{\circ}$; the maximum in the classical $E_{f}=8 U_{p}$ results is normalized to the maximum just above $30^{\circ}$ in the same quantum results (solid black curve).

The most remarkable feature of the quantum-mechanical ATD results in the left-hand panel of Fig. 11 is the distinct change in the shape of the AD for a laser intensity corresponding to the closure of the 10-photon channel (cf. the solid black curve). One sees that in contrast to the ADs for the other laser intensities (including that corresponding to the nine-photon $\mathrm{CC}$ ), the $\mathrm{AD}$ for the 10-photon CC intensity develops a large new maximum that peaks at $\theta=0^{\circ}$. Whereas a typical $\mathrm{AD}$ for an electron having a kinetic energy of $8 U_{p}$ is characterized by only one off-axis maximum, at the 10photon $\mathrm{CC}$ intensity the shape of the AD becomes similar to that for a plateau electron having a kinetic energy of $7 U_{p}$ [cf. Fig. 6(d)]. Our calculations (not shown here) predict that similar changes in the shape of the ADs occur also for higher energies on the ATD plateau (i.e., up to about $10 U_{p}$ ), with the magnitude of the change in shape diminishing with increasing electron energy.

The change in shape of the ADs for electrons detached from $\mathrm{H}^{-}$with kinetic energies near the cutoff of the ATD plateau (for a laser intensity at an even multiphoton detachment $\mathrm{CC}$ ) occur also in the ADs for electrons detached from $\mathrm{F}^{-}$(for a laser intensity at an odd multiphoton detachment $\mathrm{CC})$. In Fig. 12 we present the ADs for detachment of $\mathrm{F}^{-}$for detached electrons having the same kinetic energy, $E_{f}=8 U_{p}$, as in Fig. 11 for $\mathrm{H}^{-}$. Results are presented separately for the ADs of valence electrons having magnetic quantum number $m=0$ (left-hand panel) and $m= \pm 1$ (right-hand panel). One 


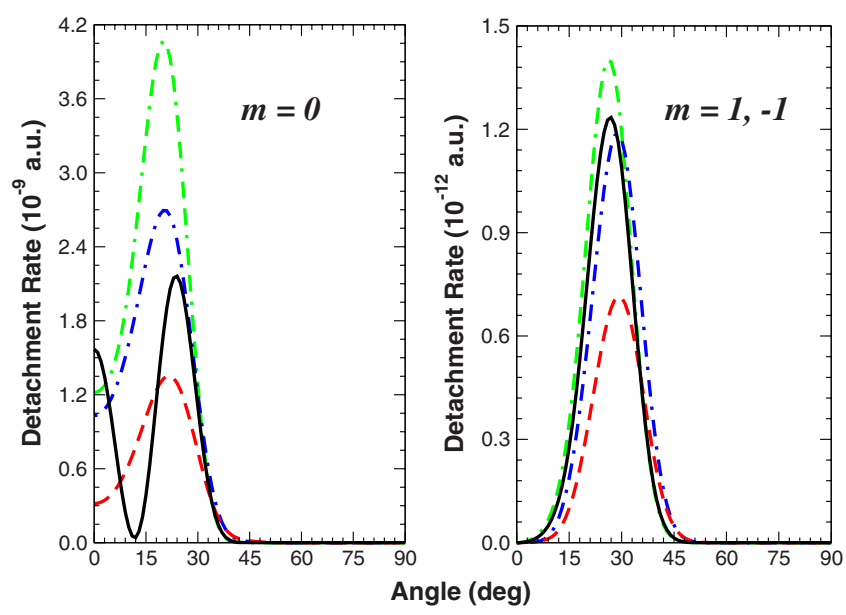

FIG. 12. (Color online) Dependence of $\mathrm{F}^{-}$ATD electron angular distributions on the initial state magnetic quantum numbers, $m=0$ and $m= \pm 1$. Results shown are for a linearly polarized laser field of frequency $\omega=0.0253$ a.u. and four values of intensity, $I$ (in units of $10^{12} \mathrm{~W} / \mathrm{cm}^{2}$ ), that span the closing of the eight- and nine-photon detachment channels: $I=6.97$ (dashed red curve, $n=33$, eightphoton CC), $I=8.1$ (dashed-dotted blue curve, $n=37$ ), $I=9.3$ (solid black curve, $n=42$, nine-photon CC), and $I=9.8$ (dashed-dasheddotted green curve, $n=45$ ), where $n$ is the number of absorbed photons needed to give the detached electrons the same final kinetic energy, $8 U_{p}$. Note the three orders of magnitude difference in the rates for $m=0$ and $m= \pm 1$.

observes the remarkable fact that for a laser intensity corresponding to the closure of the nine-photon detachment channel (cf. the solid black curves), only the AD for a detached electron having $m=0$ changes its shape. Specifically, this curve develops a significant maximum that peaks at $\theta=0^{\circ}$. This maximum at $\theta=0^{\circ}$ is absent in the $m=0$ ADs for other laser intensities as well as in the $m= \pm 1$ curves (even at the nine-photon $\mathrm{CC}$ intensity). One observes also in Fig. 12 that the rates for $m= \pm 1$ are about three orders of magnitude smaller than those for $m=0$. Hence, averaging the detachment rates over the projections of the initial angular momentum is essentially unnecessary owing to the dominant contribution of the $m=0$ results.

The classical analysis, derived for the case of only one laser-driven rescattering event of the active electron, predicts the appearance of a single, off-axis maximum in the detached electron AD, as shown in the right-hand panel of Fig. 11. Except for laser intensities corresponding to an even or odd multiphoton $\mathrm{CC}$ (depending on the symmetry of the valence electron), this classical prediction agrees qualitatively with the quantum-mechanical predictions (cf. Figs. 11, left-hand panel, and 12). More precisely, for the $8 U_{p}$ electrons the classical AD, which does not depend on the electron's initial angular momentum, shows a pronounced, very narrow maximum at around $30^{\circ}$, as measured with respect to the laser field polarization direction. Also, at $\theta=0^{\circ}$ the classical AD has a nonzero value. Both of these classical predictions agree approximately with the quantum mechanical results for laser intensities away from those corresponding to a multiphoton $\mathrm{CC}$ at which enhancement occurs. Note also that the quantum-mechanical $\mathrm{AD}$ results in the case $m= \pm 1$ have, owing to the angular momentum barrier, a zero value at $\theta$ $=0^{\circ}$ (cf. Fig. 12, right-hand panel).

With regard to the ATD plateau cutoff energies, the classical analysis presented in Sec. II C 2 gives values for these energies of $10.007 U_{p}, 9.065 U_{p}$, and $6.678 U_{p}$ for detachment angles of $\theta=0^{\circ}, \theta=20^{\circ}$, and $40^{\circ}$, respectively. These classical cutoff energies are denoted in Figs. 1 and 2 by arrows and in Fig. 3 by vertical dashed lines. One observes that these classical predictions for the dependence of the ATD plateau cutoff energies on the angle $\theta$ coincide with our quantummechanical results. Recall that the results in Figs 1, 2, and 3 are for laser intensities close to those which close particular multiphoton detachment channels, resulting in enhancements of ATD rates in the low- and mid-energy regions of the ATD plateau. As already discussed, the magnitudes of the angleintegrated ATD rates at the high-energy end of the ATD plateau, in the vicinity of the plateau cutoff, do not experience similar large enhancements with small changes of laser intensity. The present ATD rates for fixed electron detachment angle show, in addition, that the ATD plateau cutoff energy also does not change significantly with intensity in the vicinity of multiphoton transition thresholds and is explained well by a classical analysis.

\section{CONCLUSIONS}

A considerable amount of experimental and theoretical work [2-20] has been devoted to enhancements of the ATI and ATD plateau rates. As we have shown recently $[17,19]$, valuable information on the enhancement of angle-integrated ATD rates can be obtained from analyzing their variation with laser intensity over a broad range of detached electron energies. More precise information on the dynamics of ATD may be gained, however, when the angular distributions at fixed energies and the energy distributions at fixed angles are investigated. In the present paper, results of both of these kinds of investigations have been presented and discussed in detail.

Our nonperturbative Floquet study of ATD angular and energy distributions at fixed electron detachment angles (measured with respect to the direction of linear laser field polarization) for two different ionic targets, $\mathrm{H}^{-}$( $s$-electron) and $\mathrm{F}^{-}$(p-electron), has confirmed the sensitivity of these processes to the initial state symmetry of the valence electron. Under conditions for which even- or odd-multiphoton channels become closed in ATD of $\mathrm{H}^{-}$or $\mathrm{F}^{-}$respectively, we have found pronounced enhancements of the fixed-angle ATD rates over an energy region extending from the low- to the intermediate-energy region of the ATD plateau. In particular, the $\mathrm{CC}$-induced enhancement is experienced even by those electrons that are detected with nonzero transverse momenta. The energy domain over which the enhancement occurs moves towards lower energies (and thus occurs over a smaller energy range) with increasing detachment angle. For large enough detachment angle, no enhancement of the ATD rates occurs. In the case of fixed electron energy, the rate enhancement occurs over only a limited angular region, which shifts towards the polarization axis with increasing number of absorbed photons. We find also that the pro- 
nounced enhancements appear only for detachment from an initial state having orbital angular-momentum projection, $m$ $=0$. In the case of $\mathrm{F}^{-}$, initial states having $m= \pm 1$ not only have rates that are about three orders of magnitude smaller than those for $m=0$ (for the laser parameters considered in this paper), but also the rates for electrons having $m= \pm 1$ show no evidence of enhancement at laser intensities corresponding to multiphoton CCs.

For detached electron energies at the high-energy end of the ATD plateau, where no enhancement of the angleintegrated ATD rates occurs at laser intensity-induced multiphoton CCs, we have found nevertheless that the angular distributions for electrons having $m=0$ change their shape at symmetry-appropriate CCs. Specifically, for $8 U_{p}$ electrons, away from a multiphoton $\mathrm{CC}$, the quantum-mechanical ADs in the vicinity of the plateau cutoffs exhibit a single maximum at a finite nonzero angle, in agreement with the results of classical simulations of the ADs (performed similarly to the simulations of Refs. [40,41]). On the other hand, for laser intensities corresponding to a symmetry-appropriate, multiphoton CC, our quantum-mechanical ADs for electrons having $m=0$ develop a second maximum, closer to the laser polarization axis. This change in shape of the ATD ADs for energies close to the plateau cutoff therefore is concrete evidence that $\mathrm{CC}$ effects extend over a wider range of energies on the ATD plateau than expected heretofore. Moreover, this change in shape is a completely quantum-mechanical effect; it does not occur in our classical simulations. On the other hand, our classical analysis of the energy cutoff for the fixedangle ATD plateau gives predictions for these cutoffs that are in accord with our numerical quantum-mechanical results.

We note in concluding that our Yukawa-potential model calculations, although accurate (within the single-activeelectron approximation) for the description of abovethreshold detachment from negative ions, cannot account for any effects that are due to the long-range Coulomb potential that exists following ionization of neutral atoms. Also, the threshold features of multiphoton ionization channels in the presence of a Coulomb field are rather different from those in the presence of a short-range potential. Investigations of such channel-closing effects in ATI of neutral atoms is currently under investigation.

\section{ACKNOWLEDGMENTS}

This work was supported in part by the Polish Ministry of Science and Higher Education under Grant No. 1 P03B 006 28 (K.K.), and by the U.S. National Science Foundation under Grants No. PHY-0652866 (I.I.F.) and No. PHY-0601196 (A.F.S).

\section{APPENDIX: ON THE USE OF A MODEL POTENTIAL}

Our choice of the model potential (cf. Sec. II A), although physically reasonable (e.g., the screening constant $\lambda$ is of the order of $1 / r_{0}$, where $r_{0}$ is the size of the atom), is not unique. First, we can choose different combinations of $\lambda$ and $W_{0}[\mathrm{cf}$. Eq. (1)] which give the same value of the binding energy for an $s\left(\mathrm{H}^{-}\right)$or $p$ electron $\left(\mathrm{F}^{-}\right)$. Second, the effective electron-

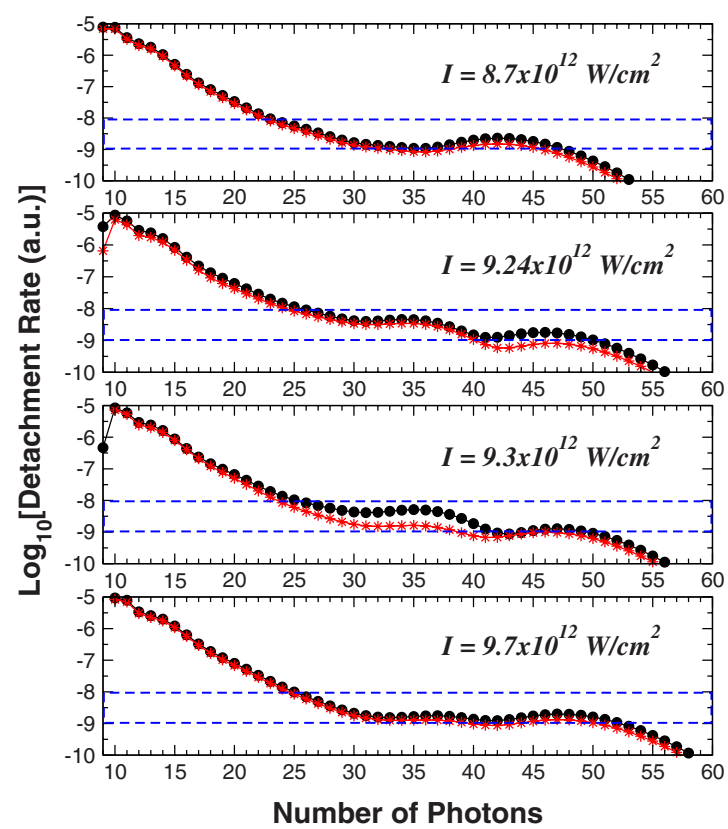

FIG. 13. (Color online) ATD spectra for $\mathrm{F}^{-}$detachment by a linearly polarized laser field of frequency $\omega=0.0253$ a.u. for four different laser intensities, denoted in each panel, that span the ninephoton CC. Results are compared for two model Yukawa potentials: the model potential introduced in Sec. II A (black filled circles) and the potential introduced in the Appendix (red stars). While for the model potential of Sec. II A the nine-photon CC is approximately at the laser intensity $I=9.3 \times 10^{12} \mathrm{~W} / \mathrm{cm}^{2}$, in the other case the same channel becomes closed at approximately $I=9.24 \times 10^{12} \mathrm{~W} / \mathrm{cm}^{2}$. All rates are averaged over the projections of the initial angular momentum, $\ell=1$. The blue dashed lines are drawn to help visualize the changing magnitudes of the detachment rates on the plateau as the laser intensity increases.

atom interaction is different for different partial waves. In particular, for $\mathrm{F}^{-}$the potential should be much less attractive in the $s$ wave, since an $s$ electron cannot be bound by the F atom. Strictly speaking, an $l$-dependent pseudopotential should be employed, if we use the single active electron approximation. However, our present formalism does not allow us to work with pseudopotentials.

To investigate the first effect, we have performed calculations of the detachment rates for a model potential describing $\mathrm{F}^{-}$, choosing $\lambda=1.1$ a.u. and $W_{0}=-5.50285$ a.u. With such a choice of parameters the binding energy of the negative ion remains the same as in Sec. II A with our original choice of $\lambda$ and $W_{0}$. Comparing the results obtained for these two potentials, we have found in both cases the same qualitative behavior of the detachment rate as a function of the laser field intensity and the photoelectron energy, including also pronounced enhancements of the ATD plateau at the closing of symmetry-appropriate multiphoton channels. As expected (and confirmed by our model potential calculations), the lowest part of the ATD spectrum is practically model independent; it is determined by the binding energy and asymptotic form of the bound state wave function, which results also in very good agreement between the model potential calculations and the theory of Ref. [38] (cf. Sec. III B). Good agreement of the detachment rates calculated for the two different 
model potentials is observed also at higher intensities and lower electron energies, where the details of the potential are similarly unimportant. However, at lower intensities and higher electron energies we observe certain differences, thus indicating the limitations of even an accurate model potential.

Concerning the main focus of this paper, we compare in Fig. 13 the threshold behavior of the $\mathrm{F}^{-}$detachment rates for two model Yukawa potentials, Eq. (1): (a) With the parameters given in Sec. II A (filled black circles); and (b) with the new parameters given above (red stars). Results are shown for laser intensities that sweep across the nine-photon ponderomotively shifted channel closing. This CC occurs approximately at the laser intensity $9.3 \times 10^{12} \mathrm{~W} / \mathrm{cm}^{2}$ in case (a) and approximately at the intensity $9.24 \times 10^{12} \mathrm{~W} / \mathrm{cm}^{2}$ in case (b), with the difference caused by a potential-dependent Stark shift of the energy levels in the laser field. For fixed laser intensity, one observes excellent agreement of the two detachment rates in the low-energy part of the ATD spectra; this agreement however deteriorates with increasing electron energy (or, the number of absorbed laser photons) for laser intensities that bring the respective channel to its closure (the second and third panels). One can see in Fig. 13 almost an order of magnitude increase of the detachment rates in the low- and mid-energy part of the ATD plateau that in both cases occurs before the nine-photon CC. While the detachment rates at the lower-energy end of the plateau increase, those at the higher-energy end of the plateau decrease. Most importantly however, the magnitude of the CC enhancement appears to be far larger than the variations in the detachment rates due to changes in the parameters of the model potential. The threshold-related enhancements are observed for any reasonable choice of model potential at only very slightly different laser field intensities. These enhancements dominate the potential dependence of the detachment rates, as shown in Fig. 13. This fact is confirmed also by our calculations with other sets of parameters (not shown) giving the same binding energy for the $e-\mathrm{F}$ system.

To see how realistically $s$-wave electrons are rescattered in our model, we must compare with $a b$ initio calculations of $e-\mathrm{F}$ scattering. We are aware of only one paper on this subject [59], which investigates ${ }^{1} P^{o}$ scattering at very low energies (below $0.027 \mathrm{eV}$ ), and so, practically, only gives the scattering length, which can be estimated as 0.38 a.u. To get a better idea about low-energy $e-\mathrm{F}$ scattering we compare it with $e-\mathrm{O}$ and $e$-Ne scattering, since basic features of electron scattering by atoms with nearly the same atomic numbers are often similar. (See, e.g., Ref. [60] concerning low-energy electron scattering by $\mathrm{S}, \mathrm{Cl}$, and $\mathrm{Ar}$ atoms.) From such a comparison, we conclude that the $e-\mathrm{F}$ scattering length should have a positive sign, since there is no RamsauerTownsend minimum in $e-\mathrm{O}$ and $e-\mathrm{Ne}$ scattering, and the corresponding scattering lengths (for ${ }^{2} P^{e}$ and ${ }^{1} S^{e}$ scattering in $\mathrm{O}$ and $\mathrm{Ne}$ ) are 0.28 a.u. and 0.214 a.u., respectively $[60,61]$. Calculations with our model potential gives an $s$-wave scattering length of 1.8 a.u., i.e., significantly higher than the above estimates. This means that $s$-wave rescattering at low energies is overestimated in our model. On the other hand, our $s$-wave scattering phase shift decreases with energy significantly slower than for $\mathrm{Ne}$ [61] resulting in the factor 4.5 difference with $\mathrm{Ne}$ for the cross sections at $E=2.2 \mathrm{eV}$ [as compared to the factor $(1.8 / 0.214)^{2}=71$ for $\left.E=0\right]$. Therefore, for higher-energy electrons our description of $s$-wave rescattering becomes more realistic.
[1] W. Becker, F. Grasbon, R. Kopold, D. B. Milošević, G. G. Paulus, and H. Walther, Adv. At., Mol., Opt. Phys. 48, 35 (2002).

[2] P. Hansch, M. A. Walker, and L. D. Van Woerkom, Phys. Rev. A 55, R2535 (1997).

[3] M. P. Hertlein, P. H. Bucksbaum, and H. G. Muller, J. Phys. B 30, L197 (1997).

[4] M. J. Nandor, M. A. Walker, L. D. Van Woerkom, and H. G. Muller, Phys. Rev. A 60, R1771 (1999).

[5] E. Cormier, D. Garzella, P. Breger, P. Agostini, G. Chériaux, and C. Leblanc, J. Phys. B 34, L9 (2000).

[6] G. G. Paulus, F. Grasbon, H. Walther, R. Kopold, and W. Becker, Phys. Rev. A 64, 021401(R) (2001).

[7] F. Grasbon, G. G. Paulus, H. Walther, P. Villoresi, G. Sansone, S. Stagira, M. Nisoli, and S. De Silvestri, Phys. Rev. Lett. 91, 173003 (2003).

[8] H. G. Muller and F. C. Kooiman, Phys. Rev. Lett. 81, 1207 (1998).

[9] H. G. Muller, Phys. Rev. A 60, 1341 (1999).

[10] R. Kopold, W. Becker, M. Kleber, and G. G. Paulus, J. Phys. B 35, 217 (2002).

[11] B. Borca, M. V. Frolov, N. L. Manakov, and A. F. Starace, Phys. Rev. Lett. 88, 193001 (2002).
[12] S. V. Popruzhenko, Ph. A. Korneev, S. P. Goreslavski, and W. Becker, Phys. Rev. Lett. 89, 023001 (2002).

[13] J. Wassaf, V. Véniard, R. Taïeb, and A. Maquet, Phys. Rev. Lett. 90, 013003 (2003).

[14] J. Wassaf, V. Véniard, R. Taïeb, and A. Maquet, Phys. Rev. A 67, 053405 (2003).

[15] K. Krajewska, I. I. Fabrikant, and A. F. Starace, Bull. Am. Phys. Soc. 51, 119 (2006).

[16] N. L. Manakov and M. V. Frolov, Pis'ma Zh. Eksp. Teor. Fiz. 83, 630 (2006) [JETP Lett. 83, 536 (2006)].

[17] K. Krajewska, I. I. Fabrikant, and A. F. Starace, Phys. Rev. A 74, 053407 (2006).

[18] R. M. Potvliege and S. Vučić, Phys. Rev. A 74, 023412 (2006).

[19] K. Krajewska, I. I. Fabrikant, and A. F. Starace, Laser Phys. 17, 368 (2007).

[20] D. B. Milošević, E. Hasovič, M. Busuladžić, A. GazibegovićBusuladžić, and W. Becker, Phys. Rev. A 76, 053410 (2007).

[21] E. P. Wigner, Phys. Rev. 73, 1002 (1948).

[22] A. I. Baz', Zh. Eksp. Teor. Fiz. 33, 923 (1957) [Sov. Phys. JETP 6, 709 (1958)].

[23] X. M. Zhao, M. S. Gulley, H. C. Bryant, C. E. M. Strauss, D. J. Funk, A. Stintz, D. C. Rislove, G. A. Kyrala, W. B. Ingalls, 
and W. A. Miller, Phys. Rev. Lett. 78, 1656 (1997).

[24] L. Praestegaard, T. Andersen, and P. Balling, Phys. Rev. A 59, R3154 (1999).

[25] R. Reichle, H. Helm, and I. Yu. Kiyan, Phys. Rev. Lett. 87, 243001 (2001).

[26] R. Reichle, I. Yu. Kiyan, and H. Helm, J. Mod. Opt. 50, 461 (2003).

[27] R. Reichle, H. Helm, and I. Yu. Kiyan, Phys. Rev. A 68, 063404 (2003).

[28] I. Sánchez, H. Bachau, and F. Martin, J. Phys. B 30, 2417 (1997).

[29] D. A. Telnov and Shih-I Chu, Phys. Rev. A 66, 063409 (2002).

[30] L. Bai, J. Zhang, X. Zhang, and Z. Xu, Phys. Rev. A 74, 025402 (2006).

[31] I. Yu. Kiyan and H. Helm, Phys. Rev. Lett. 90, 183001 (2003).

[32] B. Bergues, Y. F. Ni, H. Helm, and I. Yu. Kiyan, Phys. Rev. Lett. 95, 263002 (2005).

[33] B. Bergues, Z. Ansari, D. Hanstorp, and I. Yu. Kiyan, Phys. Rev. A 75, 063415 (2007).

[34] M. V. Frolov, N. L. Manakov, E. A. Pronin, and A. F. Starace, Phys. Rev. Lett. 91, 053003 (2003).

[35] D. B. Milošević, A. Gazibegović-Busuladžić, and W. Becker, Phys. Rev. A 68, 050702(R) (2003).

[36] M. V. Frolov, N. L. Manakov, E. A. Pronin, and A. F. Starace, J. Phys. B 36, L419 (2003).

[37] A. M. Perelomov, V. S. Popov, and M. V. Terent'ev, Zh. Eksp. Teor. Fiz. 50, 1393 (1966) [Sov. Phys. JETP 23, 924 (1966)].

[38] G. F. Gribakin and M. Yu. Kuchiev, Phys. Rev. A 55, 3760 (1997).

[39] B. Borca, M. V. Frolov, N. L. Manakov, and A. F. Starace, Phys. Rev. Lett. 87, 133001 (2001).

[40] G. G. Paulus, W. Becker, W. Nicklich, and H. Walther, J. Phys. B 27, L703 (1994).

[41] G. G. Paulus, W. Becker, and H. Walther, Phys. Rev. A 52, 4043 (1995).

[42] K. R. Lykke, K. K. Murray, and W. C. Lineberger, Phys. Rev.
A 43, 6104 (1991).

[43] C. Blondel, P. Cacciani, C. Delsart, and R. Trainham, Phys. Rev. A 40, 3698 (1989).

[44] R. Shakeshaft and X. Tang, Phys. Rev. A 36, 3193 (1987).

[45] M. Dörr, R. M. Potvliege, D. Proulx, and R. Shakeshaft, Phys. Rev. A 42, 4138 (1990).

[46] R. M. Potvliege and R. Shakeshaft, in Atoms in Intense Laser Fields, edited by M. Gavrila (Academic, Boston, 1992), p. 373.

[47] R. M. Potvliege and R. Shakeshaft, Phys. Rev. A 38, 4597 (1988).

[48] R. M. Potvliege and R. Shakeshaft, Phys. Rev. A 38, 6190 (1988).

[49] R. M. Potvliege and R. Shakeshaft, Phys. Rev. A 40, 3061 (1989).

[50] R. M. Potvliege, Comput. Phys. Commun. 114, 42 (1998).

[51] K. J. Schafer, B. Yang, L. F. DiMauro, and K. C. Kulander, Phys. Rev. Lett. 70, 1599 (1993).

[52] P. B. Corkum, Phys. Rev. Lett. 71, 1994 (1993).

[53] G. G. Paulus, W. Nicklich, H. Xu, P. Lambropoulos, and H. Walther, Phys. Rev. Lett. 72, 2851 (1994).

[54] M. Lewenstein, K. C. Kulander, K. J. Schafer, and P. H. Bucksbaum, Phys. Rev. A 51, 1495 (1995).

[55] B. Yang, K. J. Schafer, B. Walker, K. C. Kulander, P. Agostini, and L. F. DiMauro, Phys. Rev. Lett. 71, 3770 (1993).

[56] G. G. Paulus, W. Nicklich, and H. Walther, Europhys. Lett. 27, 267 (1994).

[57] A. Gazibegović-Busuladžić, D. B. Milošević, and W. Becker, Phys. Rev. A 70, 053403 (2004).

[58] E. Hasović, M. Busuladžić, A. Gazibegović-Busuladžić, D. B. Milošević, and W. Becker, Laser Phys. 17, 376 (2007).

[59] W. D. Robb and R. J. W. Henry, Phys. Rev. A 16, 2491 (1977).

[60] I. I. Fabrikant, J. Phys. B 27, 4545 (1994).

[61] T. F. O'Malley and R. W. Crompton, J. Phys. B 13, 3451 (1980). 